\title{
Complexes of metal halides with unreduced o-(imino)quinones
}

Irina V. Ershova', Irina N. Meshcheryakova', Olesya Yu. Trofimova' ${ }^{\dagger}$, Kira I. Pashanova ${ }^{\dagger}$, Kseniya V. Arsenyeva ${ }^{\dagger}$, Nadiya M. Khamaletdinova ${ }^{\dagger}$, Ivan V. Smolyaninov ${ }^{\ddagger}$, Maxim V. Arsenyev ${ }^{\dagger}$, Anton V. Cherkasov ${ }^{\dagger}$, and Alexandr V. Piskunovt,*

† G.A. Razuvaev Institute of Organometallic Chemistry of Russian Academy of Sciences, 49 Tropinina str., 603137 Nizhny Novgorod, Russia

‡ Astrakhan State Technical University, 16 Tatisheva str., 414056 Astrakhan, Russia

\section{Table of contents}

Materials and Methods

Table S1. Crystallographic data and structure refinement details for $1 \cdot \mathrm{Znl}_{2}, \quad\left[2 \cdot \mathrm{Cdl}_{2}\right]_{2}$, $\left[5 \cdot \mathrm{Cdl}_{2}\right]_{2} \cdot 2 \mathrm{CH}_{3} \mathrm{CN}, \mathbf{5} \cdot \mathrm{HgBr}_{2}, \mathbf{5} \cdot \mathrm{Hgl}_{2}$, and (imSQ $\left.{ }^{\mathrm{i}-\mathrm{Pr}}\right)_{2} \mathrm{Cd}$ (thf)

Figure S1. Molecular structure of $\left[2 \cdot \mathrm{Cdl}_{2}\right]_{2}$

Figure S2. Molecular structure of $5 \cdot \mathrm{HgBr}_{2}$

Table S2. The electronic spectroscopy data for ligands 1-5 and complexes 1-5•MX

Table S3. The calculated Metrical Oxidation States (MOS) for metal complexes with uncharged o(imino)quinones.

Table S4. Selected bond distances $(\AA)$ and angles $\left({ }^{\circ}\right)$ for (imSQ $\left.{ }^{i-P r}\right)_{2} \mathrm{Cd}($ thf $)$ 7

IR spectra of complexes $1-5 \cdot M X_{n}$ 8

NMR spectra of complexes $1-5 \cdot M X_{n}$

Cyclic voltammograms of ligands 1-5

Cyclic voltammograms of complexes $1-5 \cdot M X_{n}$ 


\section{Materials and Methods}

\section{General Considerations}

Solvents were purified using standard methods. ${ }^{1}$ Zinc, cadmium, mercury, indium, copper, $\mathrm{ZnCl}_{2}, \mathrm{ZnBr}_{2}, \mathrm{Cdl}_{2}, \mathrm{HgBr}_{2}, \mathrm{Hgl}_{2}$, and ferrocene were purchased from Aldrich. 3,6-Di-tert-butylo-benzoquinone (3,6-Q, 1), ${ }^{2}$ 4,6-di-tert-butyl- $N$-(2,6-di-methylphenyl)-o-iminobenzoquinone (imQ $\left.{ }^{\mathrm{Me}}, 2\right),{ }^{3}$ 4,6-di-tert-butyl-N-(2-methyl-6-ethylphenyl)-o-iminobenzoquinone (imQ $\left.{ }^{\mathrm{Me}, \mathrm{Et}}, 3\right){ }^{4}$ 4,6-di-tert-butyl- $N$-(2,6-di-ethylphenyl)-o-iminobenzoquinone (imQ $\left.{ }^{\mathrm{Et}}, \quad 4\right),{ }^{4}$ 4,6-di-tert-butyl- $N$ -

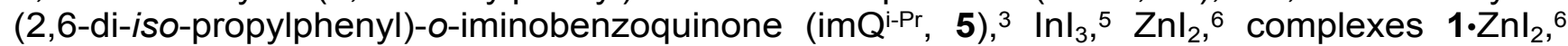

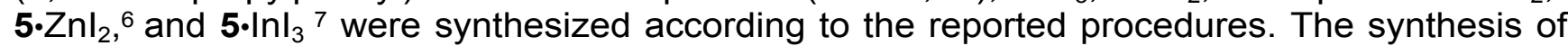
all complexes was carried out in the absence of atmospheric oxygen and moisture except for cadmium ones obtained in air.

The elemental analysis was performed on an elemental analyzer Elementar Vario EL cube. The IR spectra were recorded on an FSM 1201 spectrometer in a Nujol. ${ }^{1} \mathrm{H}$ NMR spectra were recorded on a Bruker Avance Neo $300 \mathrm{MHz}$ spectrometer using tetramethylsilane as an internal standard and $\mathrm{CDCl}_{3}$ as a solvent. The EPR spectra were obtained using a Bruker EMX spectrometer $(9.75 \mathrm{GHz})$ and simulated with the WinEPR SimFonia Software (Bruker). The electronic spectra of the complexes were recorded on a Perkin-Elmer Lambda 25 UV/Vis spectrometer (range: 220-1100 nm) at ambient temperature. Electrochemical studies were carried out using an IPC-Pro potentiostat in a three-electrode mode. The stationary platinum $(\mathrm{d}=2 \mathrm{~mm})$ disk was used as the working electrode, the platinum-flag electrode was used as the auxiliary electrode, and the $\mathrm{Ag} / \mathrm{AgCl} / \mathrm{KCl}$ (sat.) with a watertight diaphragm was used as the reference electrode. The number of electrons transferred during an electrode process was estimated relatively to ferrocene used as a standard. All measurements were carried out under argon. The samples were dissolved in pre-deaerated dichloromethane. The scan rate was $0.2 \mathrm{~V} \mathrm{~s}^{-1}$. The supporting electrolyte $0.1 \mathrm{M}\left[(\mathrm{n}-\mathrm{Bu})_{4} \mathrm{~N}\right] \mathrm{ClO}_{4}(99 \%$, "Acros") was twice recrystallized from aqueous ethanol and dried in a vacuum $(48 \mathrm{~h})$ at $50^{\circ} \mathrm{C}$. The concentration of the complexes was varied from $1 \times 10^{-3}$ to $3 \times 10^{-3} \mathrm{M}$. The redox potentials are referenced to the $\mathrm{Ag} / \mathrm{AgCl} / \mathrm{KCl}$ (sat.).

\section{Crystallography}

The X-ray data for $\mathbf{1} \cdot \mathrm{ZnI}_{2},\left[\mathbf{2} \cdot \mathrm{Cdl}_{2}\right]_{2},\left[5 \cdot \mathrm{Cdl}_{2}\right]_{2} \cdot 2 \mathrm{CH}_{3} \mathrm{CN}, \mathbf{5} \cdot \mathrm{HgBr}_{2}, \mathbf{5} \cdot \mathrm{Hgl}_{2}$, and (imSQ $\left.{ }^{\text {-Pr }}\right)_{2} \mathrm{Cd}$ (thf) were collected with Bruker Smart Apex $\left(\mathbf{1} \cdot \mathrm{Znl} \mathrm{l}_{2}\right)$, Bruker D8 Quest $\left(\left[\mathbf{5} \cdot \mathrm{Cdl}_{2}\right]_{2} \cdot 2 \mathrm{CH}_{3} \mathrm{CN}, \mathbf{5} \cdot \mathrm{HgBr}_{2}\right.$, and $\mathbf{5} \cdot \mathrm{Hgl}_{2}$, (imSQi-Pr $)_{2} \mathrm{Cd}\left(\right.$ thf)) and Rigaku OD Xcalibur $\left(\left[\mathbf{2} \cdot \mathrm{Cdl}_{2}\right]_{2}\right)$ diffractometers $\left(\mathrm{Mo}_{\mathrm{Ka}^{-}}\right.$ radiation, $\omega$-scans technique, $\lambda=0.71073 \AA$ ) using Smart, $A P E X 3^{8-9}$ and CrysAlis Pro 10 software packages. The structures were solved by direct methods and were refined by fullmatrix least squares on $F^{2}$ for all data using SHELX. ${ }^{11} S A D A B S^{12}$ and scaling algorithms implemented in CrysAlis ${ }^{\text {Pro }}$ were used to perform absorption corrections. All non-hydrogen atoms in $1 \cdot \mathrm{ZnI}_{2},\left[2 \cdot \mathrm{Cdl}_{2}\right]_{2},\left[5 \cdot \mathrm{Cdl}_{2}\right]_{2} \cdot 2 \mathrm{CH}_{3} \mathrm{CN}, \mathbf{5} \cdot \mathrm{HgBr}_{2}, \mathbf{5} \cdot \mathrm{Hgl}_{2}$, and (imSQ $\left.{ }^{\text {i-Pr }}\right)_{2} \mathrm{Cd}($ thf) were found from Fourier syntheses of electron density and were refined anisotropically. All hydrogen atoms were placed in calculated positions and were refined in the "riding" model with $U(H)_{\text {iso }}=1.2 U_{\text {eq }}$ of their parent atoms $\left(U(H)_{\text {iso }}=1.5 U_{\text {eq }}\right.$ for methyl groups $)$.

The crystallographic data and structures refinement details are given in Table S1. CCDC$2041631\left(\mathbf{1} \cdot \mathrm{ZnI}_{2}\right), 2041632\left(\left[\mathbf{2} \cdot \mathrm{Cdl}_{2}\right]_{2}\right), 2041633\left(\left[\mathbf{5} \cdot \mathrm{Cdl}_{2}\right]_{2} \cdot 2 \mathrm{CH}_{3} \mathrm{CN}\right), 2041634\left(\mathbf{5} \cdot \mathrm{HgBr}_{2}\right)$, $2041635\left(\mathbf{5} \cdot \mathrm{Hgl}_{2}\right)$, and $2084520\left(\left(\mathrm{imSQ}{ }^{\mathrm{i}-\mathrm{Pr}}\right)_{2} \mathrm{Cd}(\mathrm{thf})\right)$ contains the supplementary crystallographic data for this paper. These data are provided free of charge by The Cambridge Crystallographic Data Centre: ccdc.cam.ac.uk/structures.

\section{Computational details}

The DFT calculations were performed using the Gaussian 09 program package ${ }^{13}$ with the hybrid exchange-correlation B3LYP functional ${ }^{14}$ and standard def2-TZVP basis set for all atoms. The stationary points on the potential energy surfaces were located by full geometry optimization. The absence of imaginary frequencies suggests that the molecules are in the minimum of potential energy. 
Table S1. Crystallographic data and structure refinement details for $1 \cdot \mathrm{Znl}_{2},\left[2 \cdot \mathrm{Cdl}_{2}\right]_{2},\left[5 \cdot \mathrm{Cdl}_{2}\right]_{2} \cdot 2 \mathrm{CH}_{3} \mathrm{CN}, 5 \cdot \mathrm{HgBr}_{2}, 5 \cdot \mathrm{Hgl}_{2}$, and (imSQi-Pr) ${ }_{2} \mathrm{Cd}$ (thf)

\begin{tabular}{|c|c|c|c|c|c|c|}
\hline Compound & $1 \cdot \mathrm{ZnI}_{2}$ & {$\left[\mathbf{2} \cdot \mathrm{Cdl}_{2}\right]_{2}$} & $\left(\left[5 \cdot \mathrm{Cdl}_{2}\right]_{2} \cdot 2 \mathrm{CH}_{3} \mathrm{CN}\right.$ & $\mathbf{5} \cdot \mathrm{HgBr}_{2}$ & $5 \cdot \mathrm{Hgl}_{2}$ & $\left(\mathrm{imSQ} \mathrm{Q}^{\mathrm{i}-\mathrm{Pr}}\right)_{2} \mathrm{Cd}(\mathrm{thf})$ \\
\hline Empirical formula & $\mathrm{C}_{14} \mathrm{H}_{20} \mathrm{I}_{2} \mathrm{O}_{2} \mathrm{Zn}$ & $\mathrm{C}_{44} \mathrm{H}_{58} \mathrm{Cd}_{2} \mathrm{I}_{4} \mathrm{~N}_{2} \mathrm{O}_{2}$ & $\mathrm{C}_{56} \mathrm{H}_{80} \mathrm{Cd}_{2} \mathrm{I}_{4} \mathrm{~N}_{4} \mathrm{O}_{2}$ & $\mathrm{C}_{26} \mathrm{H}_{37} \mathrm{Br}_{2} \mathrm{HgNO}$ & $\mathrm{C}_{26} \mathrm{H}_{37} \mathrm{Hgl}_{2} \mathrm{NO}$ & $\mathrm{C}_{56} \mathrm{H}_{82} \mathrm{CdN}_{2} \mathrm{O}_{3}$ \\
\hline Formula weight & 539.47 & 1379.32 & 1573.64 & 739.97 & 833.95 & 943.63 \\
\hline Temperature $[\mathrm{K}]$ & $100.0(2)$ & $298.0(2)$ & $100.0(2)$ & 298.0(2) & $100.0(2)$ & $100(2)$ \\
\hline Crystal system & Monoclinic & Triclinic & Monoclinic & Monoclinic & Monoclinic & Triclinic \\
\hline Space group & $P 2_{1} / n$ & $P-1$ & $P 2_{1} / c$ & $P 2_{1} / n$ & $P 2_{1} / n$ & $P-1$ \\
\hline \multicolumn{7}{|l|}{ Unit cell dimensions } \\
\hline$a[\AA ̊]$ & $10.6247(6)$ & $10.2296(9)$ & $11.4506(6)$ & $12.1431(7)$ & $12.1290(5)$ & $12.7284(7)$ \\
\hline$b[\AA]$ & $9.6355(5) \mathrm{A}$ & $11.6695(6)$ & $18.4809(9)$ & $16.4602(10)$ & $16.3510(7)$ & $13.9548(7)$ \\
\hline$c[\AA]$ & $17.9078(10)$ & $11.7848(10)$ & $14.8391(8)$ & $14.4852(9)$ & $14.6598(6)$ & $16.3473(8)$ \\
\hline$\alpha\left[^{\circ}\right]$ & 90 & $106.802(6)$ & 90 & 90 & 90 & $76.744(2)$ \\
\hline$\beta\left[^{\circ}\right]$ & $94.9100(10)$ & $94.056(7)$ & $101.5046(16)$ & $90.820(2)$ & $90.9805(15)$ & $87.121(2)$ \\
\hline$\gamma\left[^{\circ}\right]$ & 90 & $104.791(6)$ & 90 & 90 & 90 & $77.666(2)$ \\
\hline$V\left[\AA^{3}\right]$ & $1826.57(17)$ & $1286.15(18)$ & $3077.1(3)$ & $2895.0(3)$ & $2906.9(2)$ & $2761.0(2)$ \\
\hline z & 4 & 1 & 2 & 4 & 4 & 2 \\
\hline$d_{\text {calc }}\left[\mathrm{g} \mathrm{cm}^{-3}\right]$ & 1.962 & 1.781 & 1.698 & 1.698 & 1.906 & 1.135 \\
\hline$\mu\left[\mathrm{mm}^{-1}\right]$ & 4.721 & 3.260 & 2.737 & 8.093 & 7.435 & 0.435 \\
\hline$F_{000}$ & 1024 & 660 & 1536 & 1432 & 1576 & 1008 \\
\hline Crystal dimensions [mm $\left.\mathrm{mm}^{3}\right]$ & $0.33 \times 0.11 \times 0.08$ & $0.30 \times 0.15 \times 0.08$ & $0.28 \times 0.10 \times 0.03$ & $0.15 \times 0.14 \times 0.06$ & $0.34 \times 0.20 \times 0.09$ & $0.25 \times 0.09 \times 0.04$ \\
\hline$\theta$ range for data collection $\left[{ }^{\circ}\right]$ & 2.15-25.09 & $3.17-26.02$ & $2.12-27.16$ & 2.08-27.19 & 2.49-30.14 & $2.47-25.11$ \\
\hline Reflections collected & 14126 & 17355 & 39578 & 45862 & 46823 & 32273 \\
\hline Independent reflections $\left(R_{\text {int }}\right)$ & $3238\left(R_{\text {int }}=0.0378\right)$ & $5078\left(R_{\text {int }}=0.0553\right)$ & $6803\left(R_{\text {int }}=0.0606\right)$ & $6413\left(R_{\text {int }}=0.0263\right)$ & $8563\left(R_{\text {int }}=0.0371\right)$ & $9693\left(R_{\text {int }}=0.0368\right)$ \\
\hline Completeness to $\theta[\%]$ & 99.5 & 99.7 & 99.9 & 99.8 & 99.7 & 98.3 \\
\hline Data/restraints/parameters & 3238 / 0 / 178 & $5078 / 222 / 252$ & $6803 / 0 / 318$ & 6413 / $307 / 303$ & 8563 / 0 / 290 & 9693 / 637 / 645 \\
\hline & $R_{1}=0.0331$ & $R_{1}=0.0631$ & $R_{1}=0.0338$ & $R_{1}=0.0347$ & $R_{1}=0.0214$ & $R_{1}=0.0421$ \\
\hline Final $R$ indices $[l>2 \sigma(I)]$ & $w R_{2}=0.0852$ & $w R_{2}=0.1494$ & $w R_{2}=0.0505$ & $w R_{2}=0.0783$ & $w R_{2}=0.0426$ & $w R_{2}=0.0794$ \\
\hline \multirow[t]{2}{*}{ Final $R$ indices (all data) } & $R_{1}=0.0378$ & $R_{1}=0.1017$ & $R_{1}=0.0584$ & $R_{1}=0.0491$ & $R_{1}=0.0271$ & $R_{1}=0.0534$ \\
\hline & $w R_{2}=0.0886$ & $w R_{2}=0.1763$ & $w R_{2}=0.0560$ & $w R_{2}=0.0845$ & $w R_{2}=0.0442$ & $w R_{2}=0.0834$ \\
\hline$S\left(F^{2}\right)$ & 1.046 & 1.067 & 1.044 & 1.030 & 1.058 & 1.066 \\
\hline Largest diff. peak and hole [e $\AA^{-3}$ ] & $2.39 /-1.38$ & $1.82 /-1.62$ & $0.99 /-0.86$ & $0.81 /-1.40$ & $0.79 /-1.25$ & $0.52 /-1.09$ \\
\hline
\end{tabular}




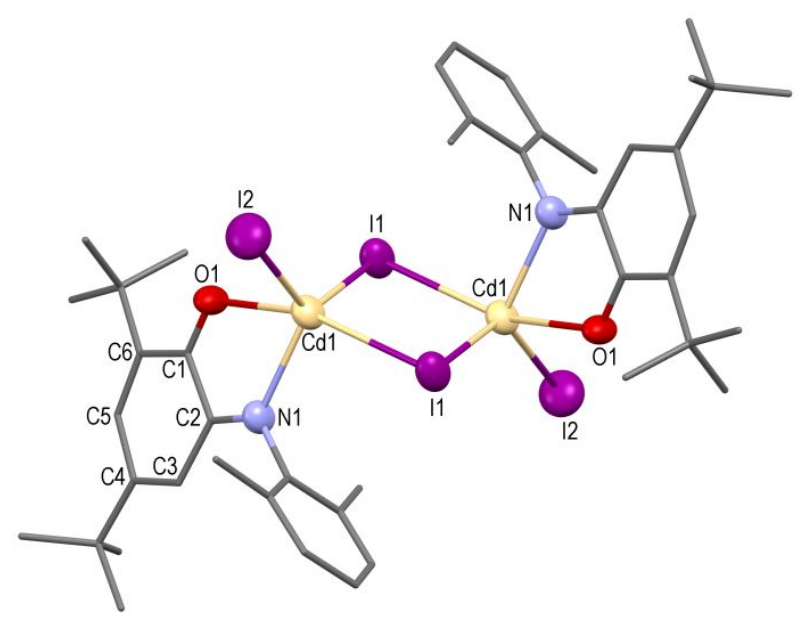

Figure S1. Molecular structure of $\left[\mathbf{2} \cdot \mathrm{Cdl}_{2}\right]_{2}$. Thermal ellipsoids of heteroatoms are drawn at the $50 \%$ probability level. Hydrogen atoms are omitted.

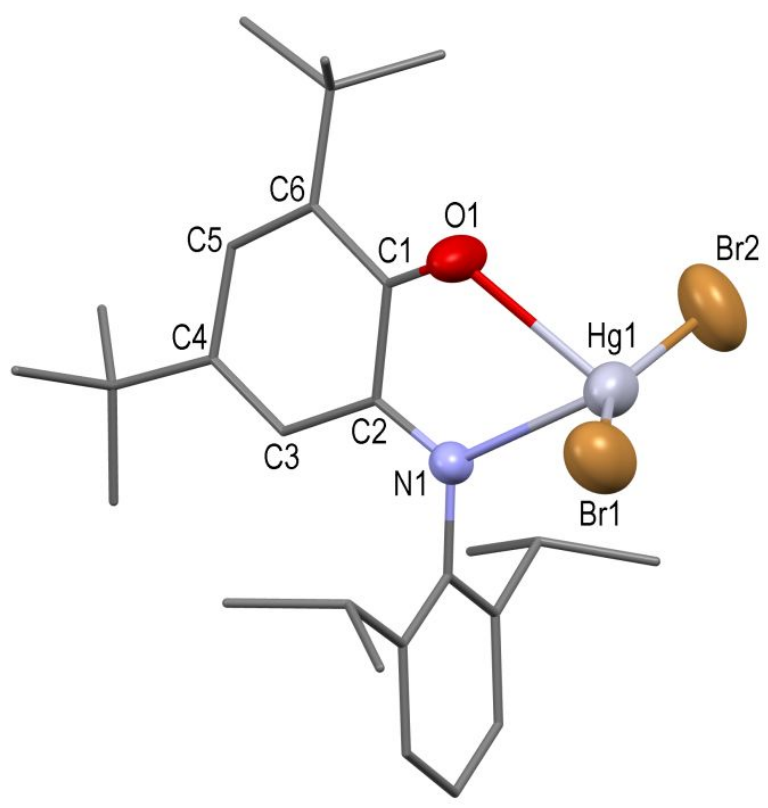

Figure S2. Molecular structure of $\mathbf{5} \cdot \mathrm{HgBr}_{2}$. Thermal ellipsoids of heteroatoms are drawn at the $50 \%$ probability level. Hydrogen atoms are omitted. 
Table S2. The electronic spectroscopy data for ligands 1-5 and complexes 1-5•MX

\begin{tabular}{|c|c|c|c|c|c|c|c|}
\hline & $\lambda, \mathrm{nm}(\varepsilon)$ & & $\lambda, \mathrm{nm}(\varepsilon)$ & \multicolumn{3}{|c|}{$\lambda, \mathrm{nm}(\varepsilon)$} & $\lambda, \mathrm{nm}(\varepsilon)$ \\
\hline 1 & $\begin{array}{l}414(2232) \\
585(70)\end{array}$ & $1 \cdot \mathrm{ZnI}_{2}$ & $\begin{array}{l}332(1630) \\
429(1672) \\
561(968)\end{array}$ & $1 \cdot \mathrm{nBr}_{2}$ & $\begin{array}{l}424(1264) \\
544(645)\end{array}$ & & \\
\hline 2 & $\begin{array}{l}389(3026) \\
500(892)\end{array}$ & $2 \cdot \mathrm{ZnI}_{2}$ & $\begin{array}{l}294(4884) \\
480(3076) \\
\end{array}$ & $2 \cdot \mathrm{Cdl}_{2}$ & $\begin{array}{l}295(3520) \\
457(2094) \\
\end{array}$ & $\left.2 \cdot \ln \right|_{3}$ & $\begin{array}{l}269 \text { (11195) } \\
487(2684)\end{array}$ \\
\hline 3 & $\begin{array}{l}392(3078) \\
500(1117) \\
\end{array}$ & $\left.3 \cdot \cdot \mathrm{ZnI}\right|_{2}$ & $\begin{array}{l}293(5810) \\
481(3181) \\
\end{array}$ & $3 \cdot \mathrm{Cdl}_{2}$ & $\begin{array}{l}296(2677) \\
457(1616) \\
\end{array}$ & $\left.3 \cdot \ln \right|_{3}$ & $\begin{array}{l}267(13022) \\
492(3158)\end{array}$ \\
\hline 4 & $\begin{array}{l}391(3323) \\
500(674)\end{array}$ & $4 \cdot \mathrm{ZnI}_{2}$ & $\begin{array}{l}290(5236) \\
481(2990)\end{array}$ & $4 \cdot \mathrm{Cdl}_{2}$ & $\begin{array}{l}294(3380) \\
458(1846) \\
\end{array}$ & $\left.4 \cdot \ln \right|_{3}$ & $\begin{array}{l}273(12174) \\
487(2932) \\
\end{array}$ \\
\hline & 395 (2952) & $5 \cdot \mathrm{ZnI}_{2}$ & $\begin{array}{l}304(7279) \\
402(3133) \\
481(3092)\end{array}$ & $5 \cdot \mathrm{Cdl}_{2}$ & $\begin{array}{l}302(7559) \\
405(2530) \\
474(2332)\end{array}$ & $\left.5 \cdot \ln \right|_{3}$ & $\begin{array}{l}292(17659) \\
406(2727) \\
483(2627)\end{array}$ \\
\hline 5 & $500(550)$ & $5 \cdot \mathrm{HgBr}_{2}$ & $\begin{array}{l}395(4365) \\
522(828)\end{array}$ & $5 \cdot \mathrm{HgI}_{2}$ & $\begin{array}{l}263(15871) \\
395(4423) \\
524(808)\end{array}$ & & \\
\hline
\end{tabular}


Table S3. The calculated Metrical Oxidation States (MOS) for metal complexes with uncharged o-(imino)benzoquinones (synthesized since 2013), calculated with the use of Seth Brown's calculator ${ }^{15}$

\begin{tabular}{|c|c|c|c|}
\hline Complex & MOS & e.s. d. & Ref. \\
\hline \multicolumn{4}{|c|}{ o-Benzoquinone complexes } \\
\hline$\left[\mathrm{Mn}(\mathrm{hfac})_{2}(3,6-\mathrm{Q})\right]$ & $\begin{array}{l}-0.08 \\
-0.07\end{array}$ & $\begin{array}{l}0.11 \\
0.15 \\
\end{array}$ & 16 \\
\hline$\left[\mathrm{Co}(\mathrm{hfac})_{2}(3,6-\mathrm{Q})\right]$ & $\begin{array}{l}-0.14 \\
-0.26\end{array}$ & $\begin{array}{l}0.15 \\
0.10\end{array}$ & 16 \\
\hline$\left[\mathrm{Co}(\mathrm{hfac})_{2}(3,5-\mathrm{Q})\right]$ & $\begin{array}{l}-0.13 \\
-0.08 \\
\end{array}$ & $\begin{array}{l}0.06 \\
0.07 \\
\end{array}$ & 16 \\
\hline$(3,6-\mathrm{Q}) \mathrm{ZnI} 2$ & -0.13 & 0.12 & this work \\
\hline \multicolumn{4}{|c|}{ o-Iminobenzoquinone complexes } \\
\hline$\left[\mathrm{Ru}^{\mathrm{II}}\left(\mathrm{L}^{\mathrm{SPh}}{ }_{\mathrm{IQ}}\right)\left(\mathrm{PPh}_{3}\right) \mathrm{Cl}_{2}\right]$ & -0.88 & 0.07 & 17 \\
\hline$\left(o-\mathrm{NO}_{2}-\mathrm{OPh}-\mathrm{imQ}\right)_{2} \mathrm{CuCl}_{2}$ & $\begin{array}{l}0.20 \\
0.20 \\
\end{array}$ & $\begin{array}{l}0.07 \\
0.07 \\
\end{array}$ & 18 \\
\hline$(\mathrm{H} \text {-imQ })_{2} \mathrm{CuCl}_{2}$ & $\begin{array}{l}0.20 \\
0.20 \\
\end{array}$ & $\begin{array}{l}0.06 \\
0.06 \\
\end{array}$ & 18 \\
\hline$(\mathrm{Ph}-\mathrm{imQ})_{2} \mathrm{CuCl}$ & $\begin{array}{l}0.07 \\
0.07 \\
\end{array}$ & $\begin{array}{l}0.04 \\
0.04 \\
\end{array}$ & 19 \\
\hline$\left[(\mathrm{Ph}-\mathrm{imQ})_{2} \mathrm{Cu}\right]^{+}\left[\mathrm{SbF}_{6}\right]^{-}$ & $\begin{array}{l}0.07 \\
0.07\end{array}$ & $\begin{array}{l}0.08 \\
0.08\end{array}$ & 19 \\
\hline$(\operatorname{dipp}-\mathrm{imQ})_{2} \mathrm{Ndl}_{3}$ & $\begin{array}{l}0.01 \\
0.01 \\
\end{array}$ & $\begin{array}{l}0.08 \\
0.04 \\
\end{array}$ & 20 \\
\hline$(\operatorname{dipp}-\mathrm{imQ})_{2} \mathrm{Dyl}_{3}$ & $\begin{array}{l}0.17 \\
0.05\end{array}$ & $\begin{array}{l}0.06 \\
0.05\end{array}$ & 21 \\
\hline$(\operatorname{dipp}-\mathrm{imQ})_{2} \mathrm{Hol}_{3}$ & $\begin{array}{l}0.04 \\
0.14 \\
\end{array}$ & $\begin{array}{l}0.05 \\
0.05\end{array}$ & 21 \\
\hline $\mathrm{UO}_{2}\left(\operatorname{dipp}\right.$-imQ)(OTf) ${ }_{2} \mathrm{THF}$ & 0.03 & 0.06 & 22 \\
\hline$\left(\operatorname{dipp}\right.$-imQ)UCl${ }_{4}(\mathrm{THF})_{2}$ & 0.04 & 0.06 & 22 \\
\hline (dipp-imQ) $\mathrm{HgBr}_{2}$ & 0.19 & 0.06 & this work \\
\hline$(\operatorname{dipp}-\mathrm{imQ}) \mathrm{Hgl}_{2}$ & 0.22 & 0.05 & this work \\
\hline$\left[(\operatorname{dipp}-\mathrm{imQ}) \mathrm{Cdl}_{2}\right]_{2} \cdot 2 \mathrm{CH}_{3} \mathrm{CN}$ & 0.18 & 0.05 & this work \\
\hline$\left[(\mathrm{dmp}-\mathrm{imQ}) \mathrm{Cdl}_{2}\right]_{2}$ & 0.15 & 0.01 & this work \\
\hline
\end{tabular}

where 3,6-Q-3,6-di-tert-butyl-o-benzoquinone;

3,5-Q - 3,5-di-tert-butyl-o-benzoquinone;

dipp-imQ - 4,6-di-tert-butyl-N-(2,6-di-iso-propylphenyl)-o-iminobenzoquinone;

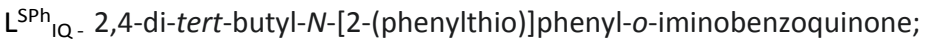

o- $\mathrm{NO}_{2}$-OPh-imQ - 2,4-di-tert-butyl-6-((2-(2-nitrophenoxy)phenyl)imino)cyclohexa-2,4-dienone;

H-imQ - 4,6-di-tert-butyl- $N$-phenyl-o-iminobenzoquinone;

Ph-imQ - 6-([1,1'-biphenyl]-2-ylimino)-2,4-di-tert-butylcyclohexa-2,4-dienone;

dmp-imQ - 4,6-di-tert-butyl- $N$-(2,6-dimethylphenyl)-o-iminobenzoquinone; 


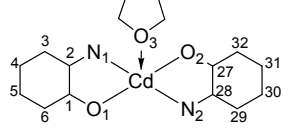

\begin{tabular}{lc|lc}
\hline \multicolumn{2}{c|}{ Bond distances, $\AA$} & \multicolumn{2}{|c}{ Angles, deg. } \\
\hline $\mathrm{Cd}-\mathrm{O}_{1}$ & $2.250(2)$ & $\mathrm{N}_{1}-\mathrm{Cd}_{1}-\mathrm{N}_{2}$ & $139.35(8)$ \\
$\mathrm{Cd}-\mathrm{N}_{1}$ & $2.195(2)$ & $\mathrm{N}_{1}-\mathrm{Cd}_{1}-\mathrm{O}_{2}$ & $109.10(7)$ \\
$\mathrm{Cd}-\mathrm{O}_{2}$ & $2.236(2)$ & $\mathrm{N}_{2}-\mathrm{Cd}_{1}-\mathrm{O}_{2}$ & $74.79(7)$ \\
$\mathrm{Cd}-\mathrm{N}_{2}$ & $2.201(2)$ & $\mathrm{N}_{1}-\mathrm{Cd}_{1}-\mathrm{O}_{1}$ & $74.60(7)$ \\
$\mathrm{Cd}_{2}-\mathrm{O}_{3}$ & $2.34(2)$ & $\mathrm{N}_{2}-\mathrm{Cd}_{1}-\mathrm{O}_{1}$ & $100.38(7)$ \\
$\mathrm{O}_{1}-\mathrm{C}_{1}$ & $1.285(3)$ & $\mathrm{O}_{2}-\mathrm{Cd}_{1}-\mathrm{O}_{1}$ & $175.17(7)$ \\
$\mathrm{N}_{1}-\mathrm{C}_{2}$ & $1.338(3)$ & $\mathrm{N}_{1}-\mathrm{Cd}_{1}-\mathrm{O}_{3}$ & $109.8(14)$ \\
$\mathrm{O}_{2}-\mathrm{C}_{27}$ & $1.282(3)$ & $\mathrm{N}_{2}-\mathrm{Cd}_{1}-\mathrm{O}_{3}$ & $110.5(15)$ \\
$\mathrm{N}_{2}-\mathrm{C}_{28}$ & $1.332(3)$ & $\mathrm{O}_{2}-\mathrm{Cd}_{1}-\mathrm{O}_{3}$ & $91.2(8)$ \\
$\mathrm{C}_{1}-\mathrm{C}_{2}$ & $1.471(4)$ & $\mathrm{O}_{1}-\mathrm{Cd}_{1}-\mathrm{O}_{3}$ & $90.4(9)$ \\
$\mathrm{C}_{2}-\mathrm{C}_{3}$ & $1.421(4)$ & & \\
$\mathrm{C}_{3}-\mathrm{C}_{4}$ & $1.364(4)$ & & \\
$\mathrm{C}_{4}-\mathrm{C}_{5}$ & $1.433(4)$ & & \\
$\mathrm{C}_{5}-\mathrm{C}_{6}$ & $1.371(4)$ & & \\
$\mathrm{C}_{1}-\mathrm{C}_{6}$ & $1.437(4)$ & & \\
$\mathrm{C}_{27}-\mathrm{C}_{28}$ & $1.469(4)$ & & \\
$\mathrm{C}_{28}-\mathrm{C}_{29}$ & $1.420(4)$ & & \\
$\mathrm{C}_{29}-\mathrm{C}_{30}$ & $1.365(4)$ & & \\
$\mathrm{C}_{30}-\mathrm{C}_{31}$ & $1.429(4)$ & & \\
$\mathrm{C}_{31}-\mathrm{C}_{32}$ & $1.369(4)$ & & \\
$\mathrm{C}_{27}-\mathrm{C}_{32}$ & $1.443(4)$ & & \\
\hline
\end{tabular}


IR spectra of complexes $1-5 \cdot \mathrm{MX}_{\mathrm{n}}$
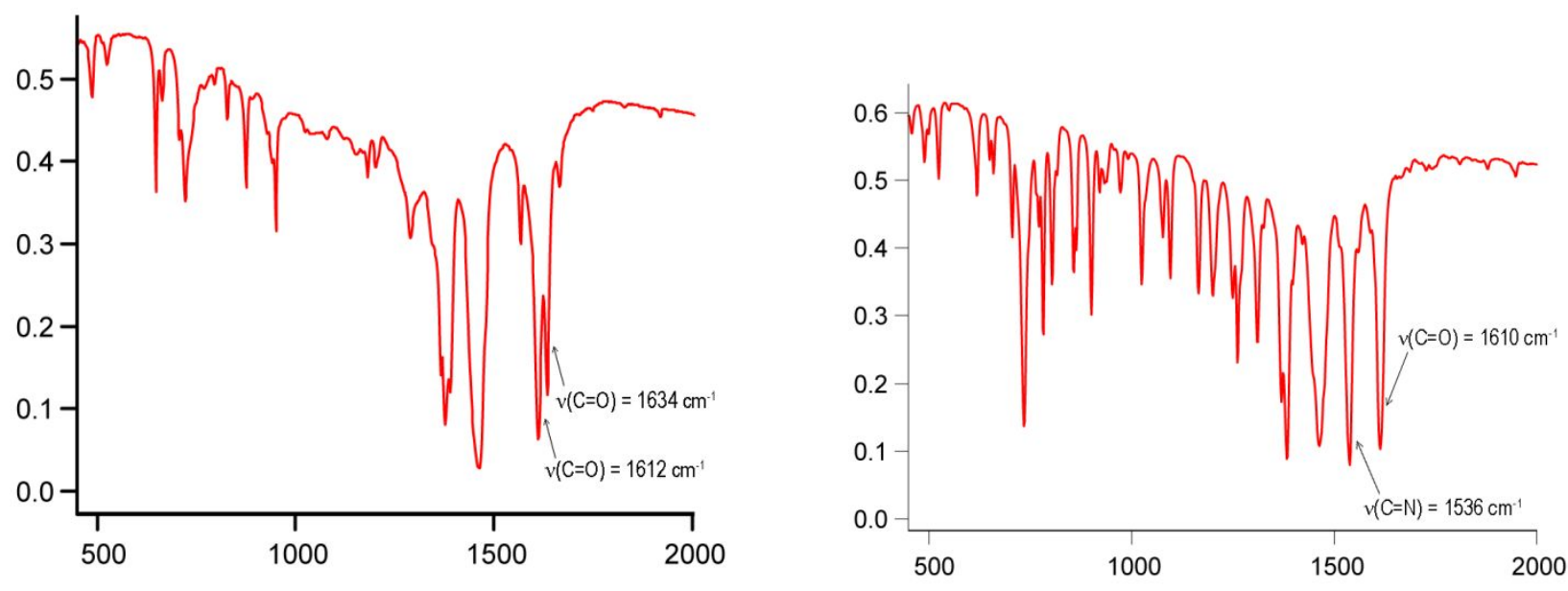

IR spectrum of $1 \cdot \mathrm{ZnCl}_{2}$

IR spectrum of $\mathbf{2} \cdot \mathrm{ZnI}_{2}$
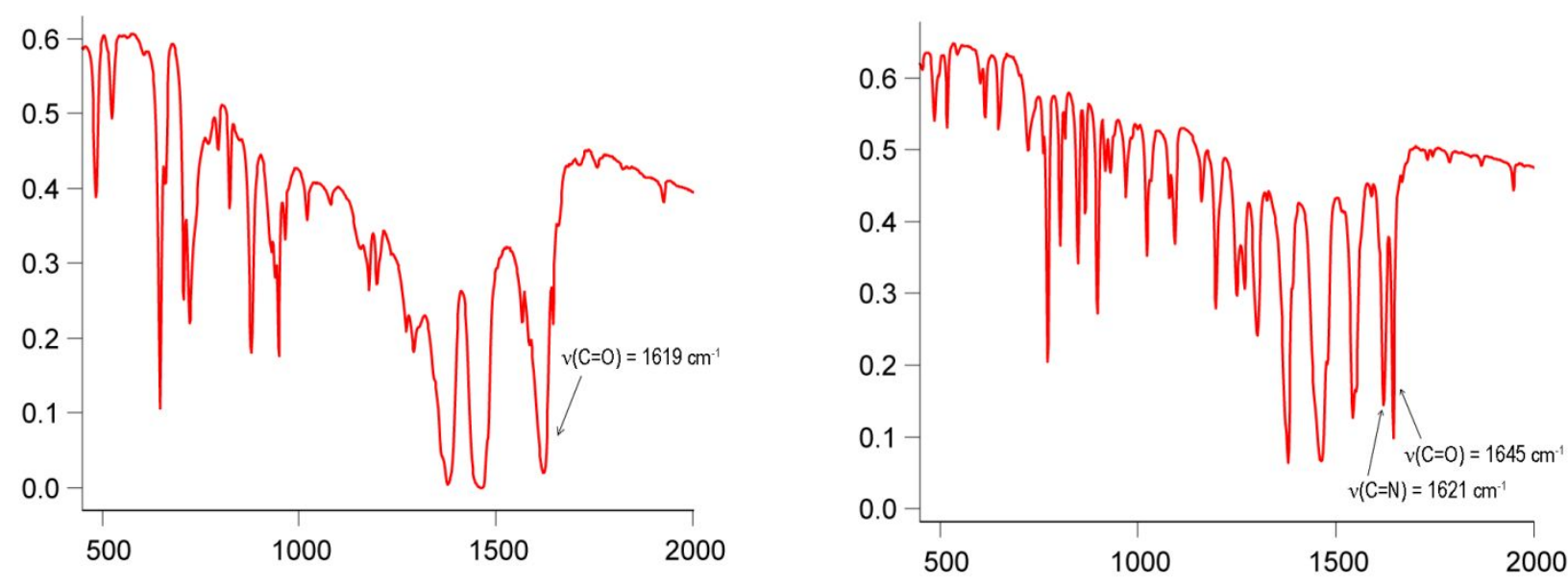

IR spectrum of $1 \cdot \mathrm{ZnBr}_{2}$

IR spectrum of $\mathbf{2} \cdot \mathrm{Cdl}_{2}$

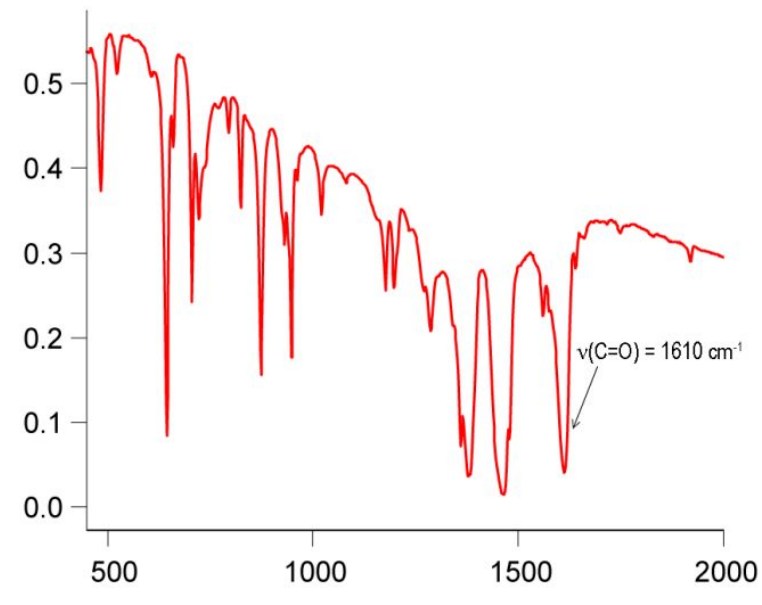

IR spectrum of $\mathbf{1} \cdot \mathrm{ZnI}_{2}$

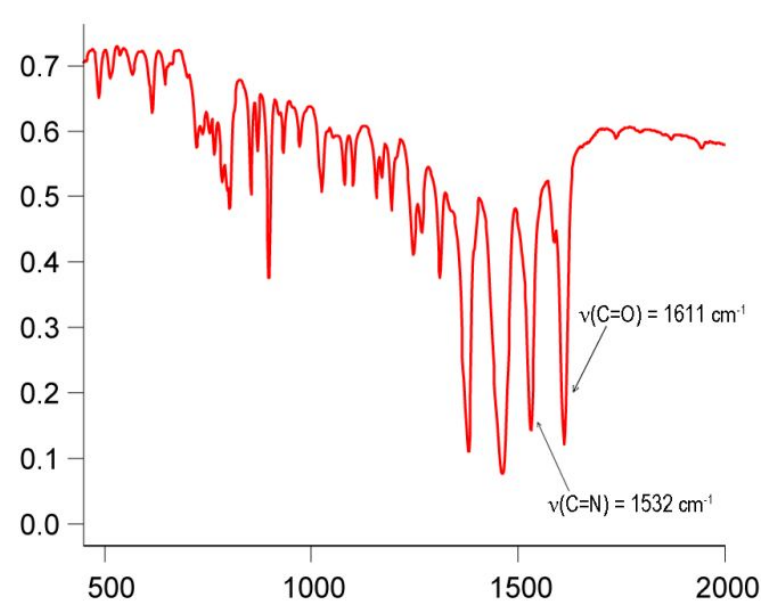

IR spectrum of $\left.2 \cdot \operatorname{lnl}\right|_{3}$ 

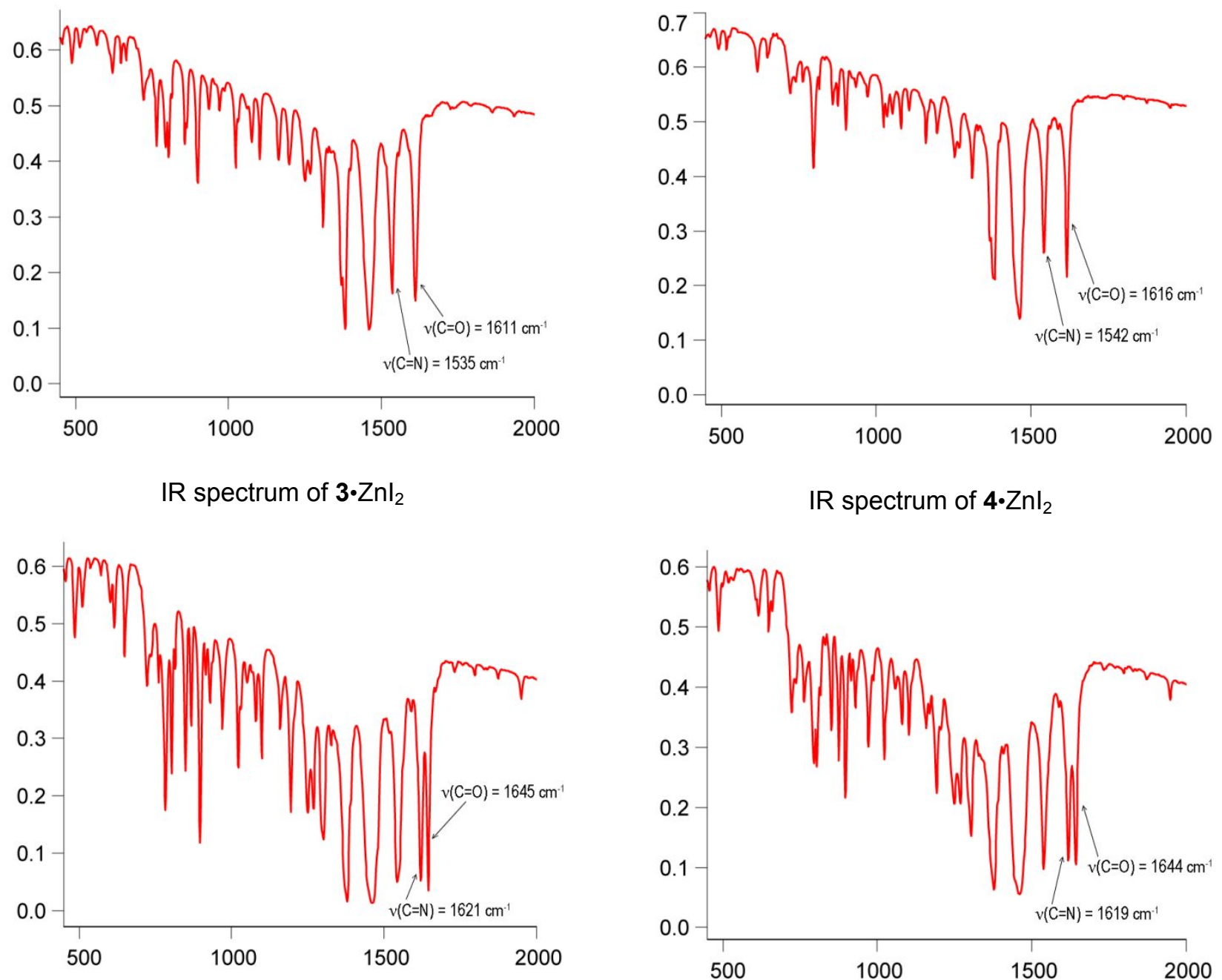

IR spectrum of $4 \cdot \mathrm{Znl}_{2}$

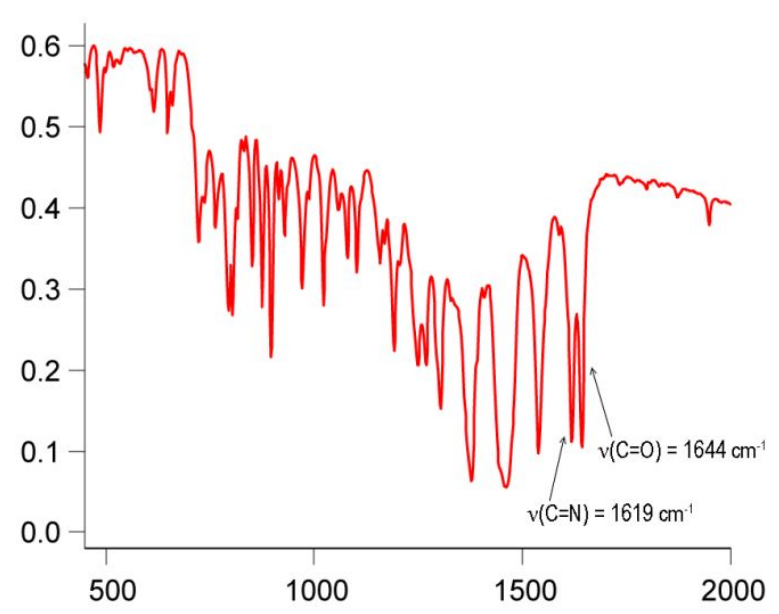

IR spectrum of $3 \cdot \mathrm{Cdl}_{2}$

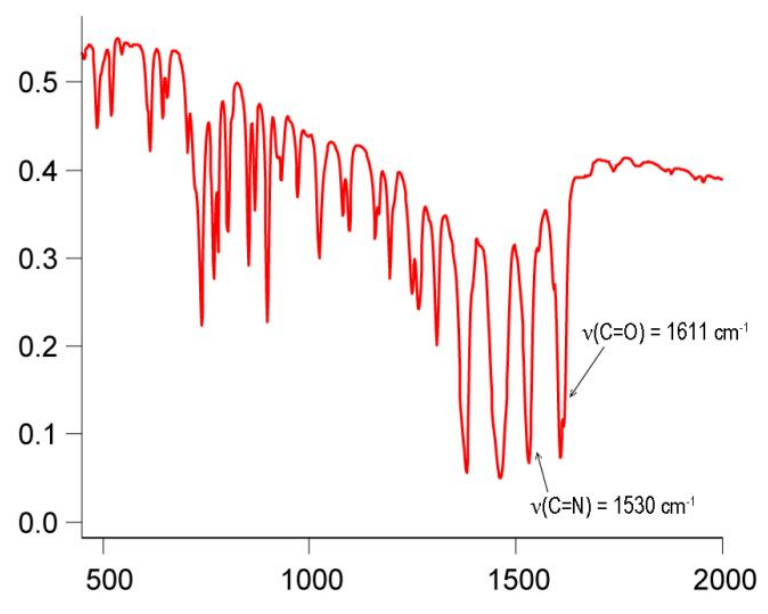

IR spectrum of $3 \cdot \mid \mathrm{Il}_{3}$

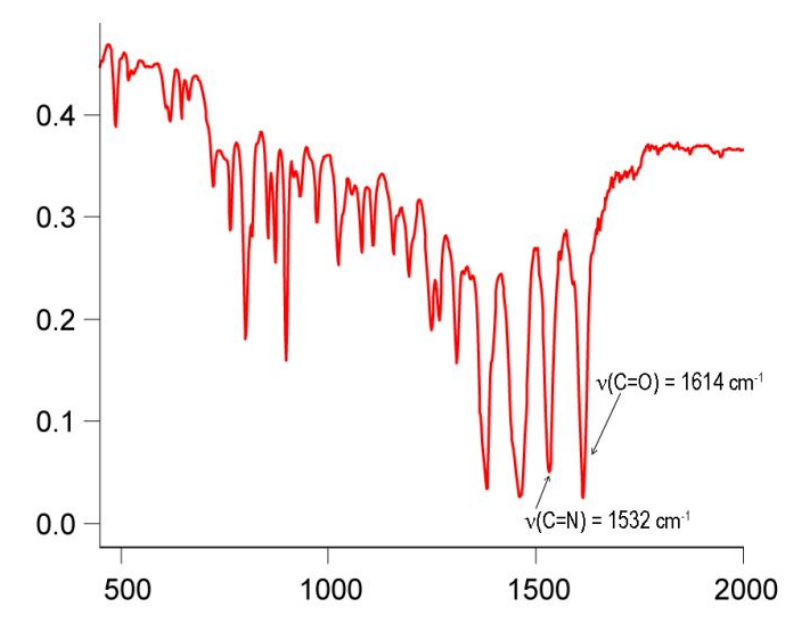

IR spectrum of $\mathbf{4} \cdot \operatorname{Inl}_{3}$ 

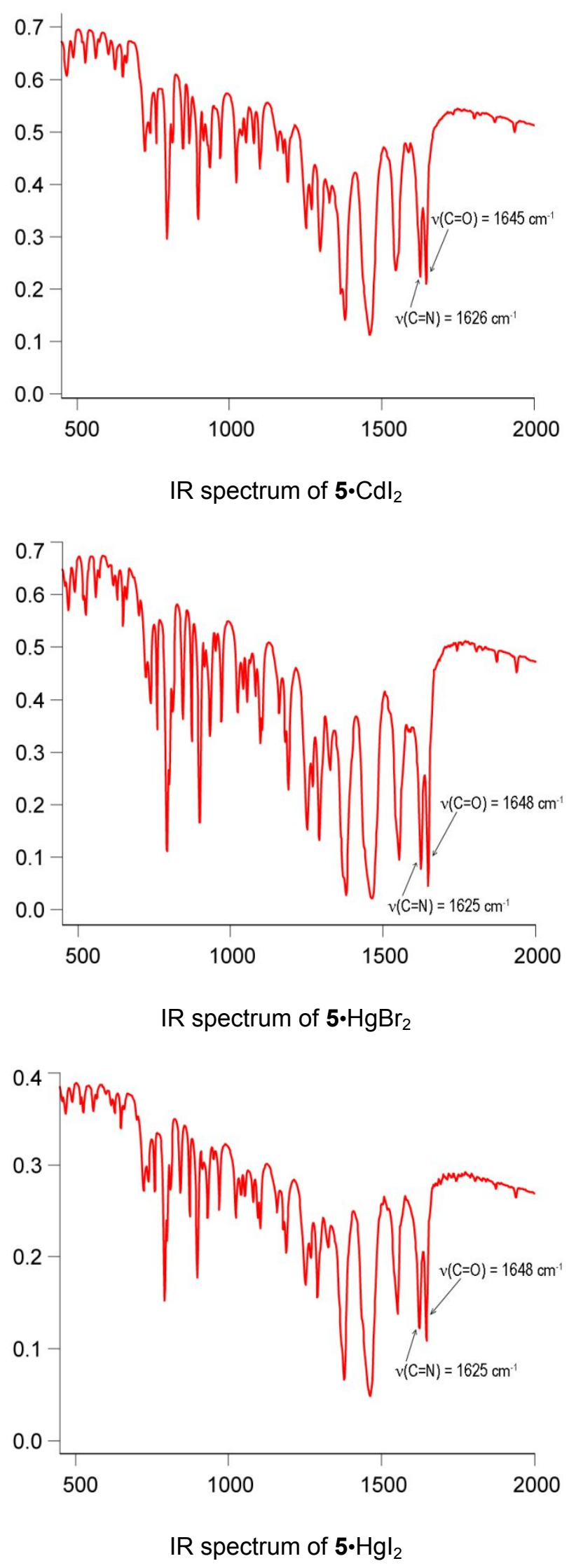
${ }^{1} \mathrm{H}$ NMR spectra of complexes $1-5 \cdot M X_{n}$

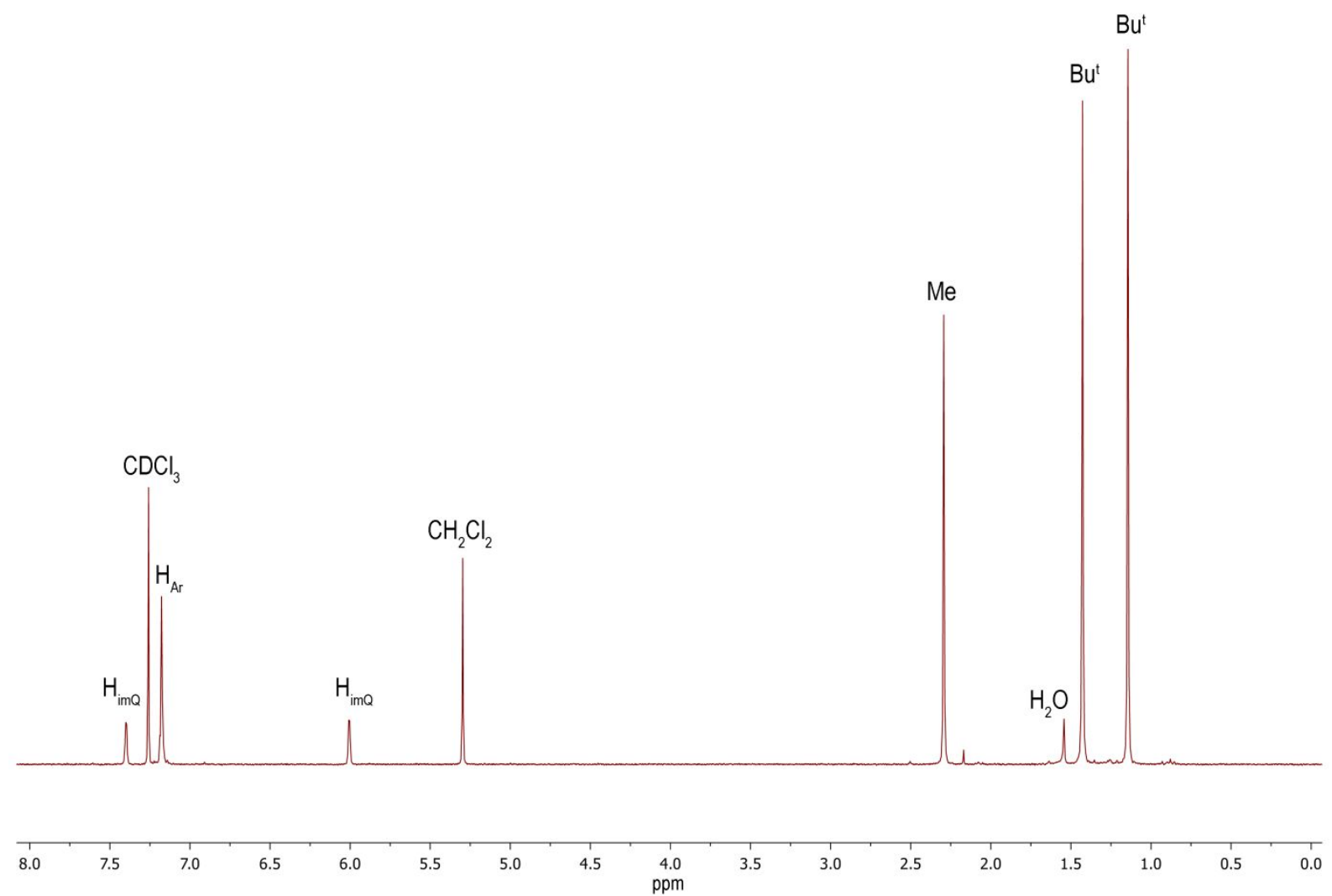

${ }^{1} \mathrm{H}$ NMR spectrum of $\mathbf{2} \cdot \mathrm{Znl}_{2}$

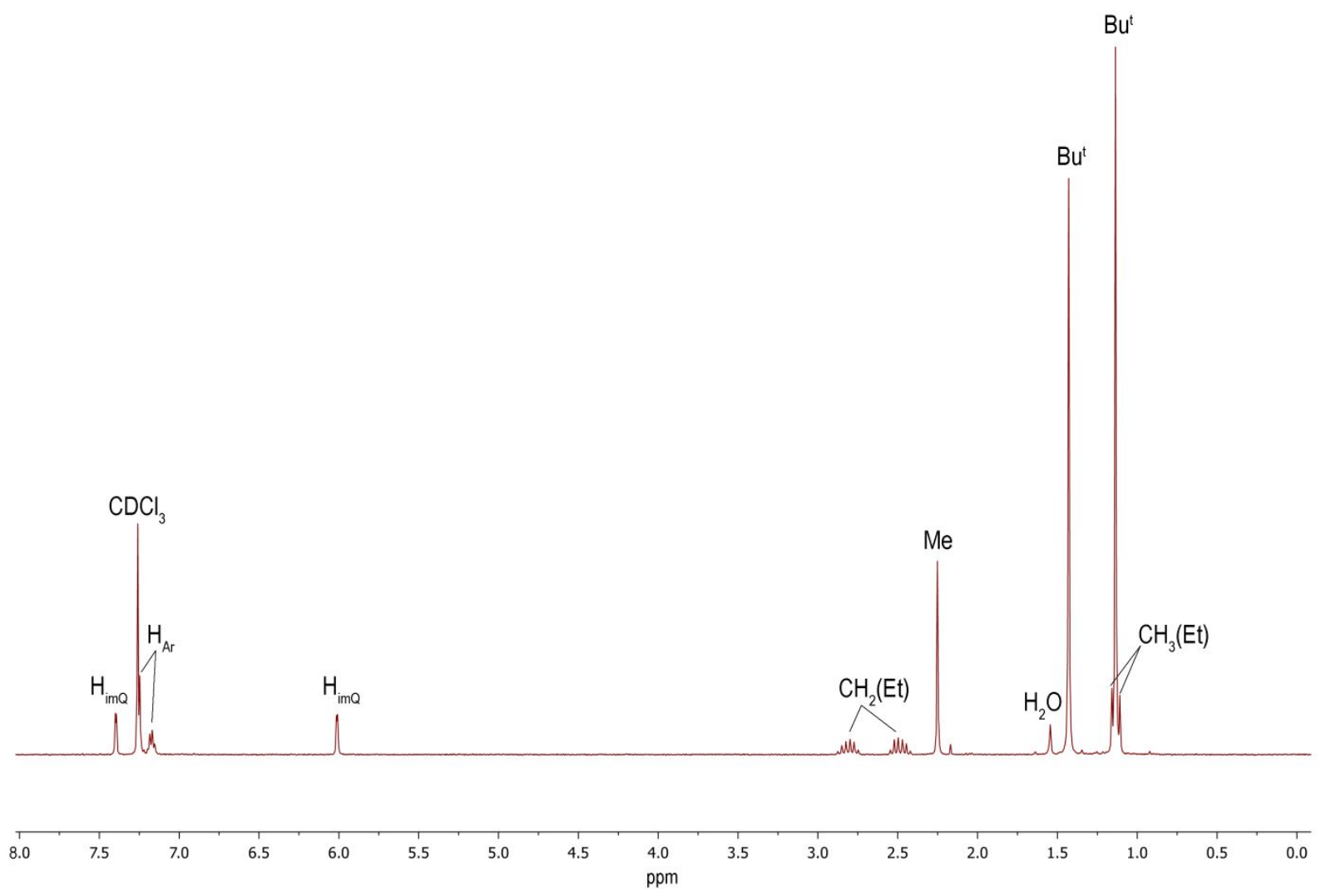

${ }^{1} \mathrm{H}$ NMR spectrum of $3 \cdot \mathrm{Znl}_{2}$ 


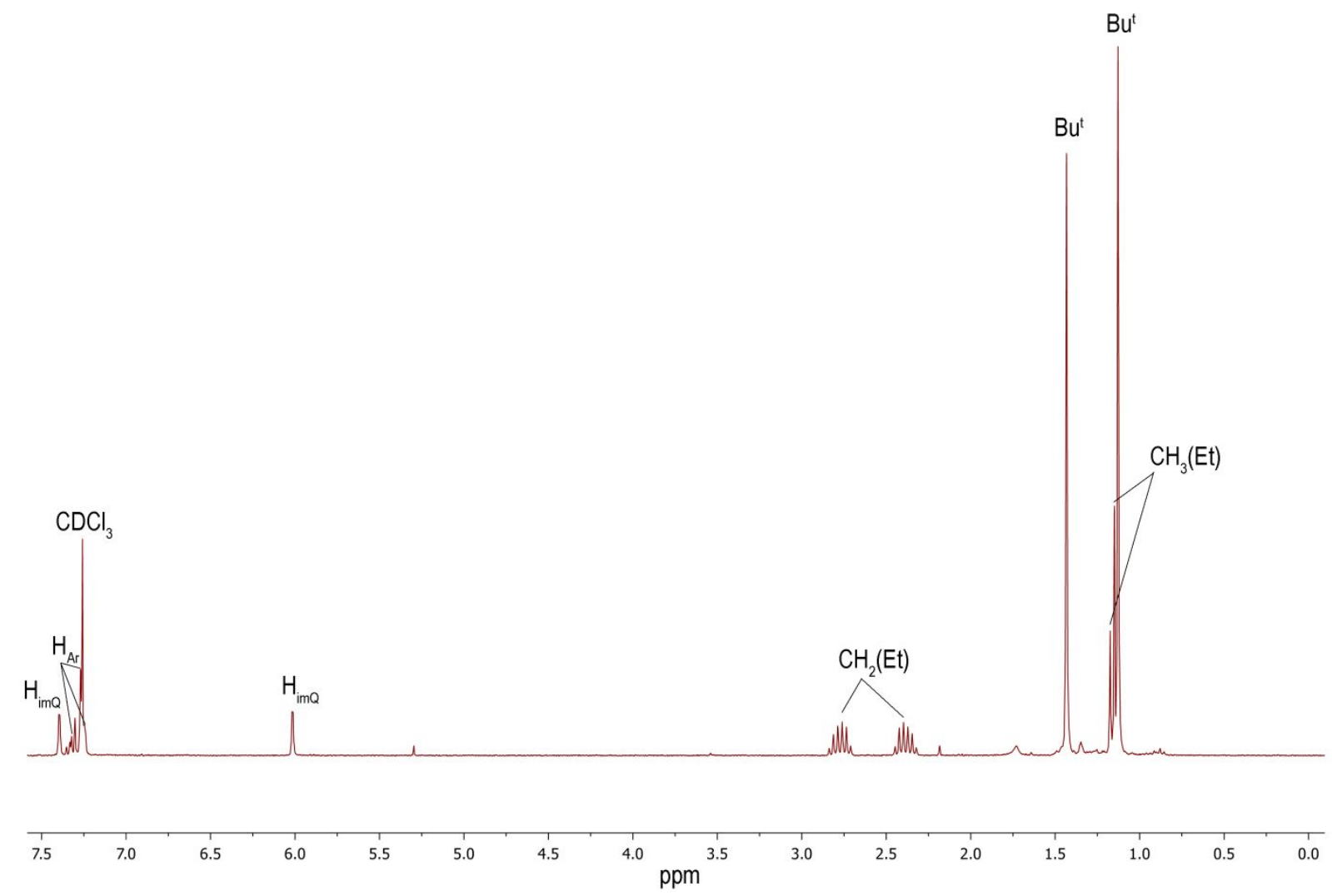

${ }^{1} \mathrm{H}$ NMR spectrum of $4 \cdot \mathrm{ZnI}_{2}$

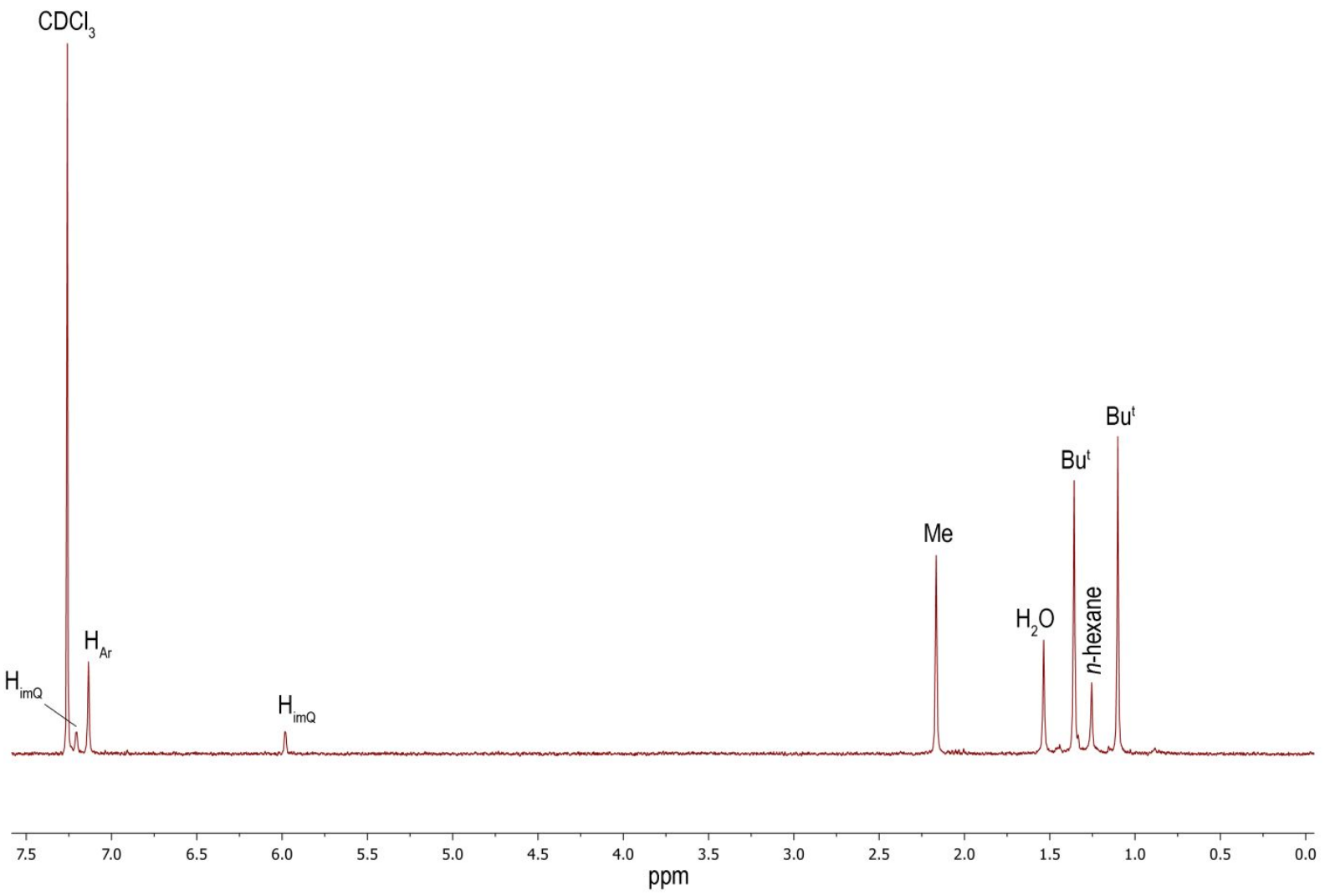

${ }^{1} \mathrm{H}$ NMR spectrum of $\mathbf{2} \cdot \mathrm{Cdl}_{2}$ 


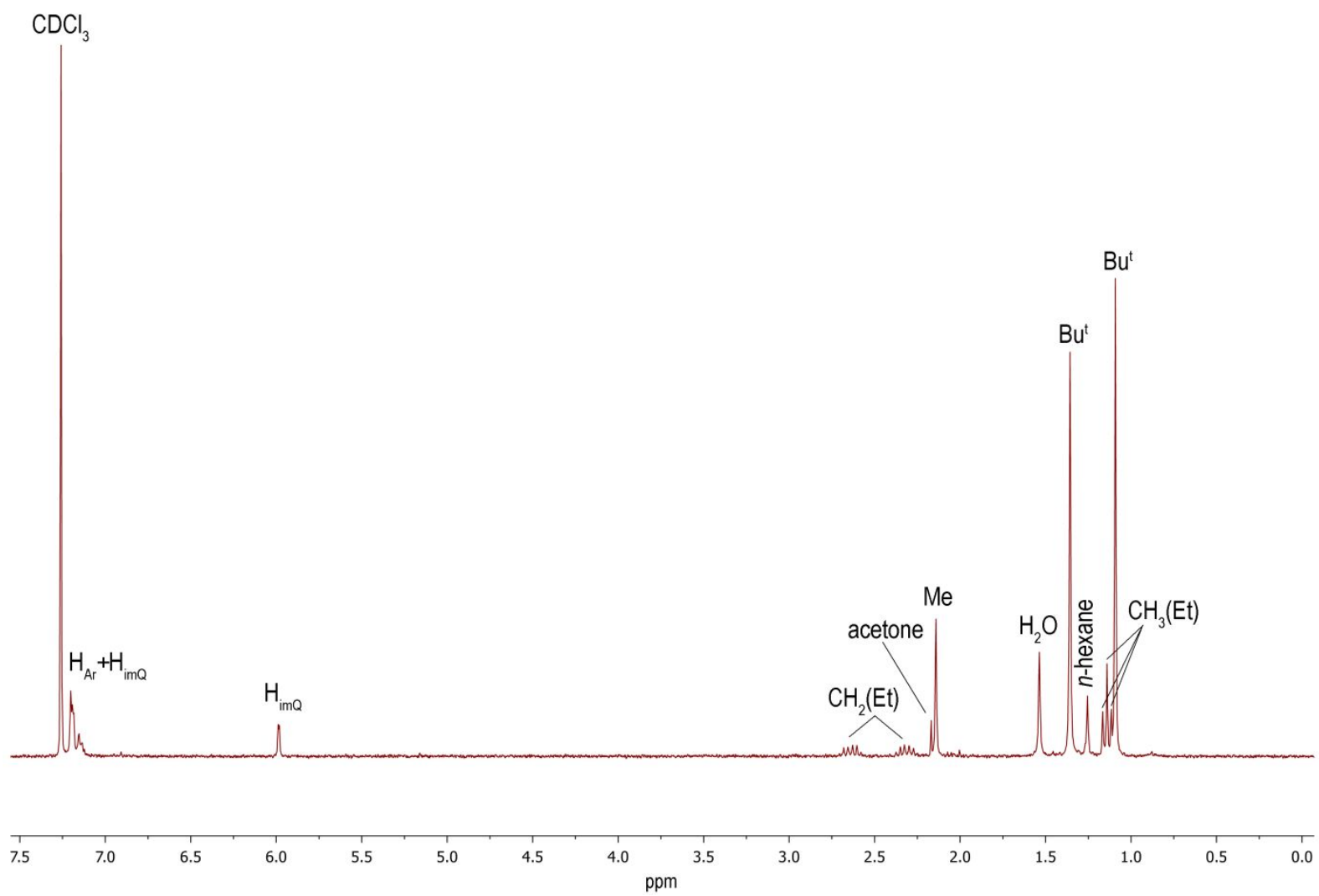

${ }^{1} \mathrm{H}$ NMR spectrum of $3 \cdot \mathrm{Cdl}_{2}$

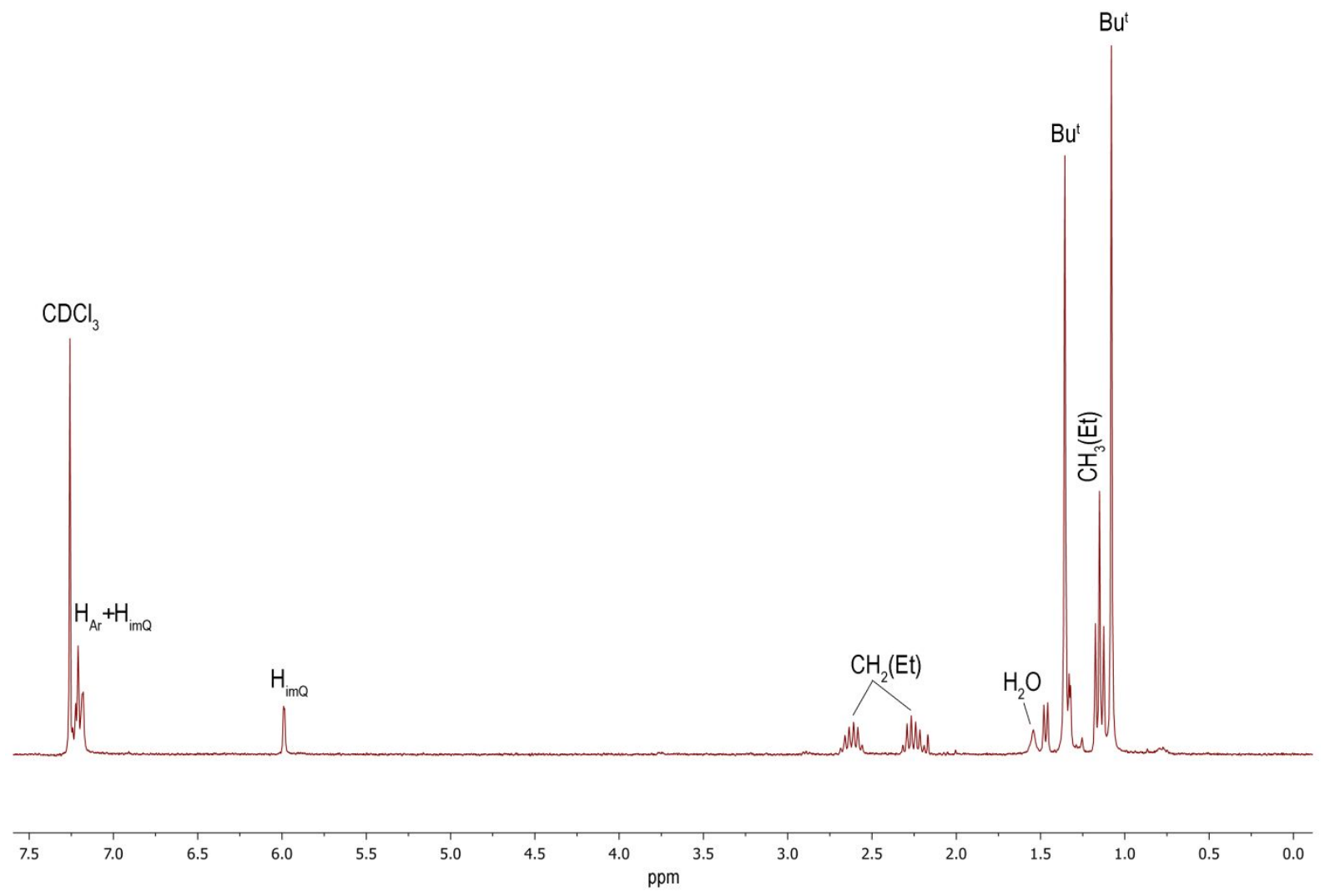

${ }^{1} \mathrm{H}$ NMR spectrum of $4 \cdot \mathrm{Cdl}_{2}$ 


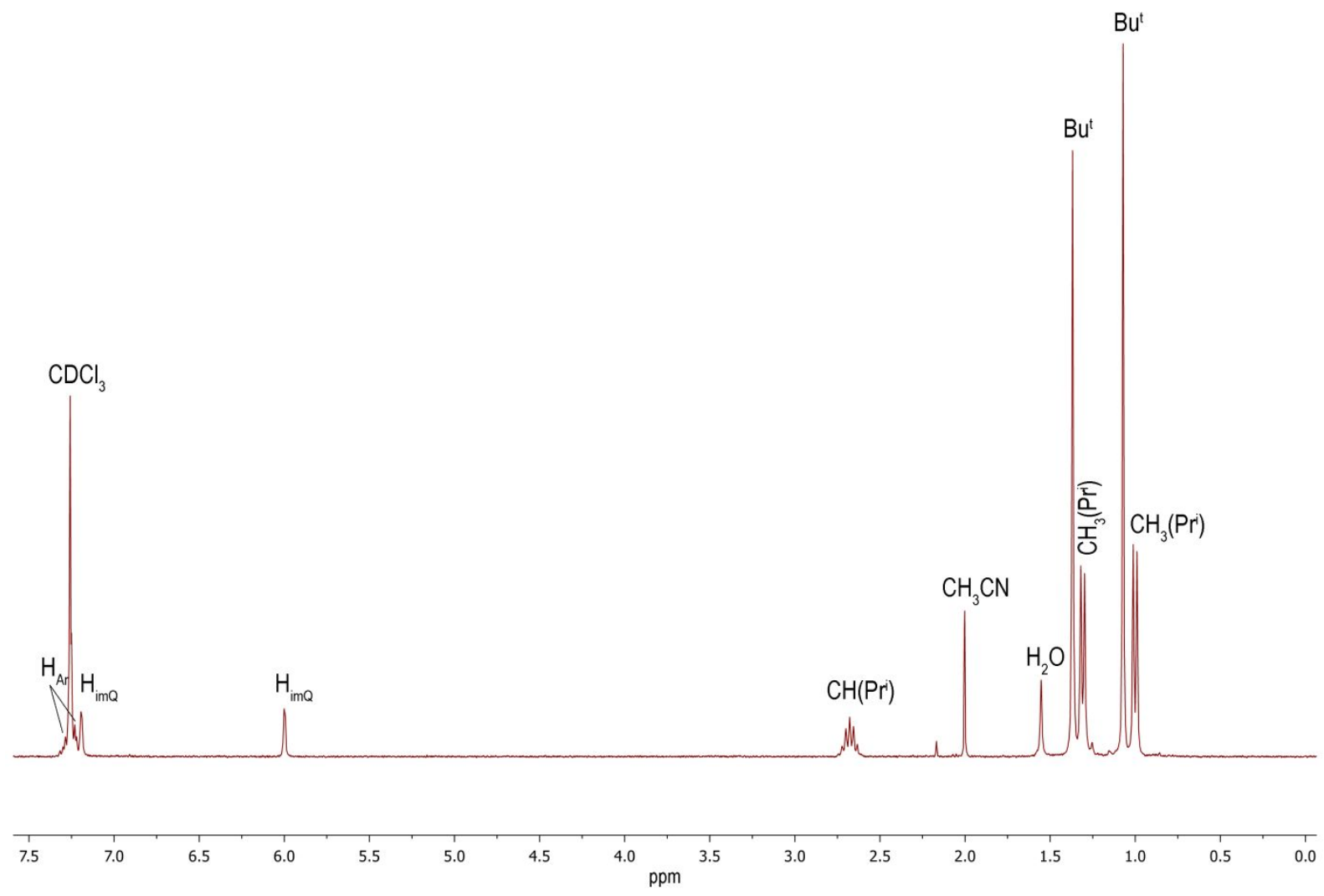

${ }^{1} \mathrm{H}$ NMR spectrum of $\mathbf{5} \cdot \mathrm{Cdl}_{2}$

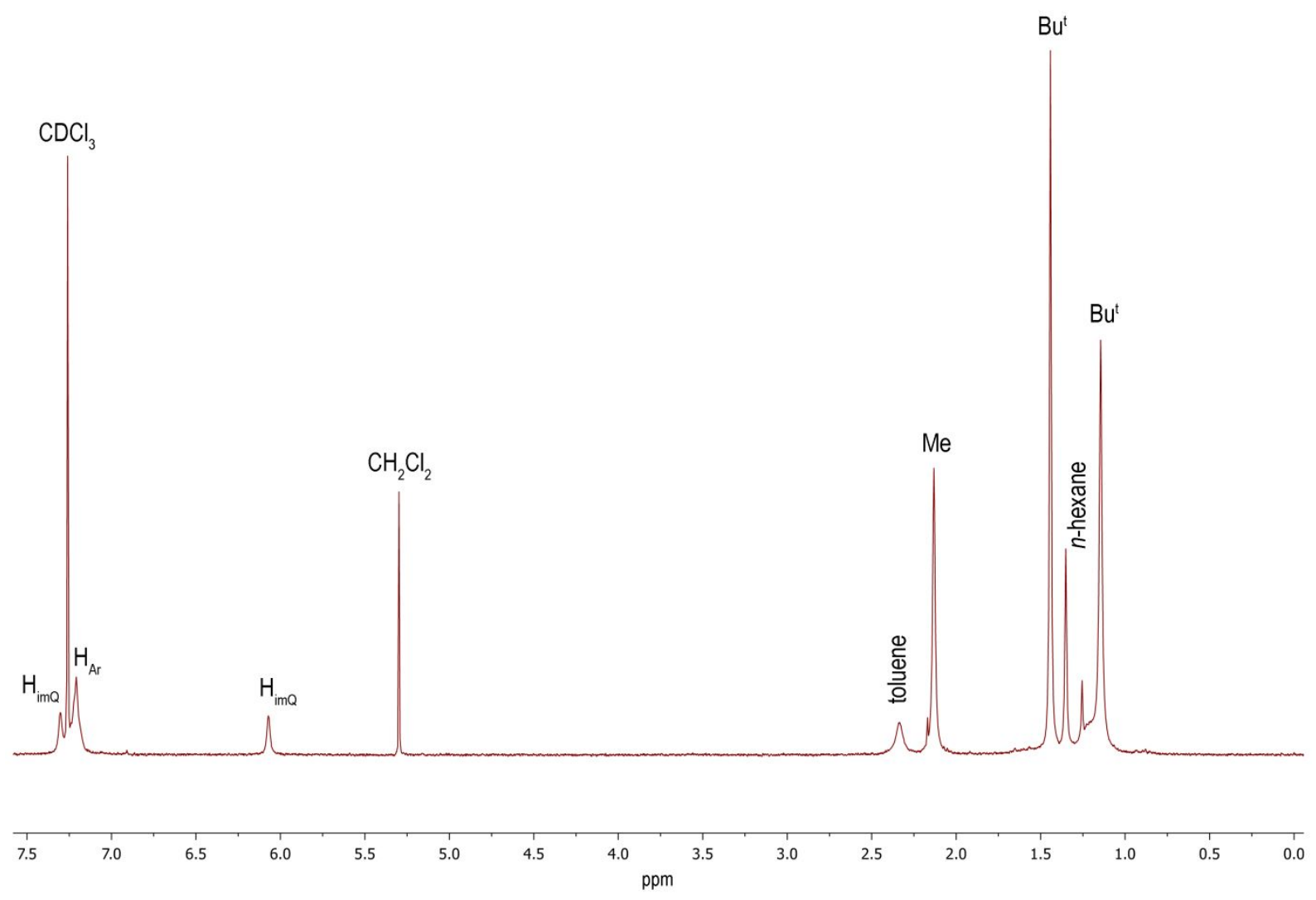

${ }^{1} \mathrm{H}$ NMR spectrum of $2 \cdot \operatorname{Inl}_{3}$ 

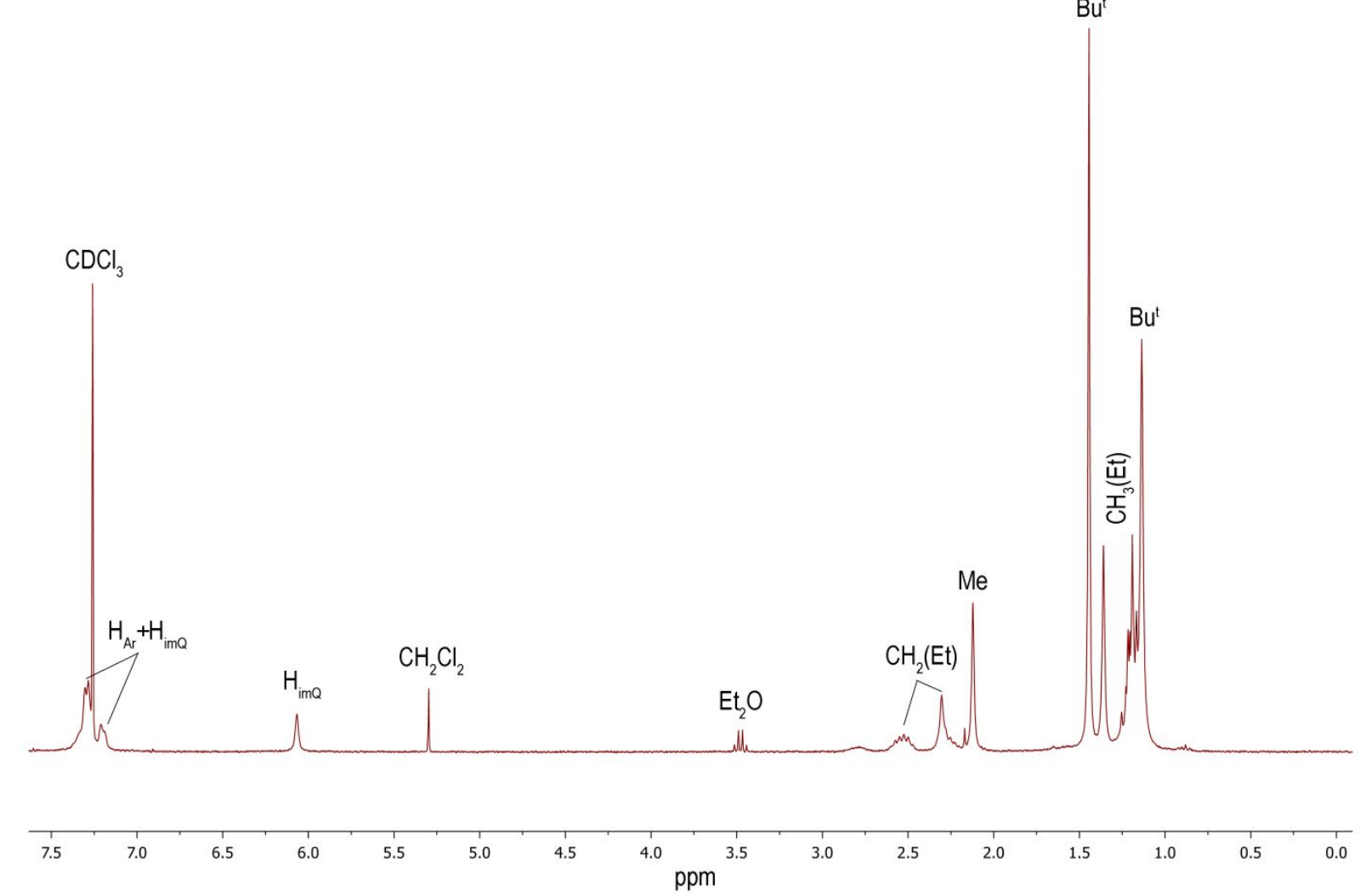

${ }^{1} \mathrm{H}$ NMR spectrum of $\mathbf{3} \cdot \mathrm{InI}_{3}$

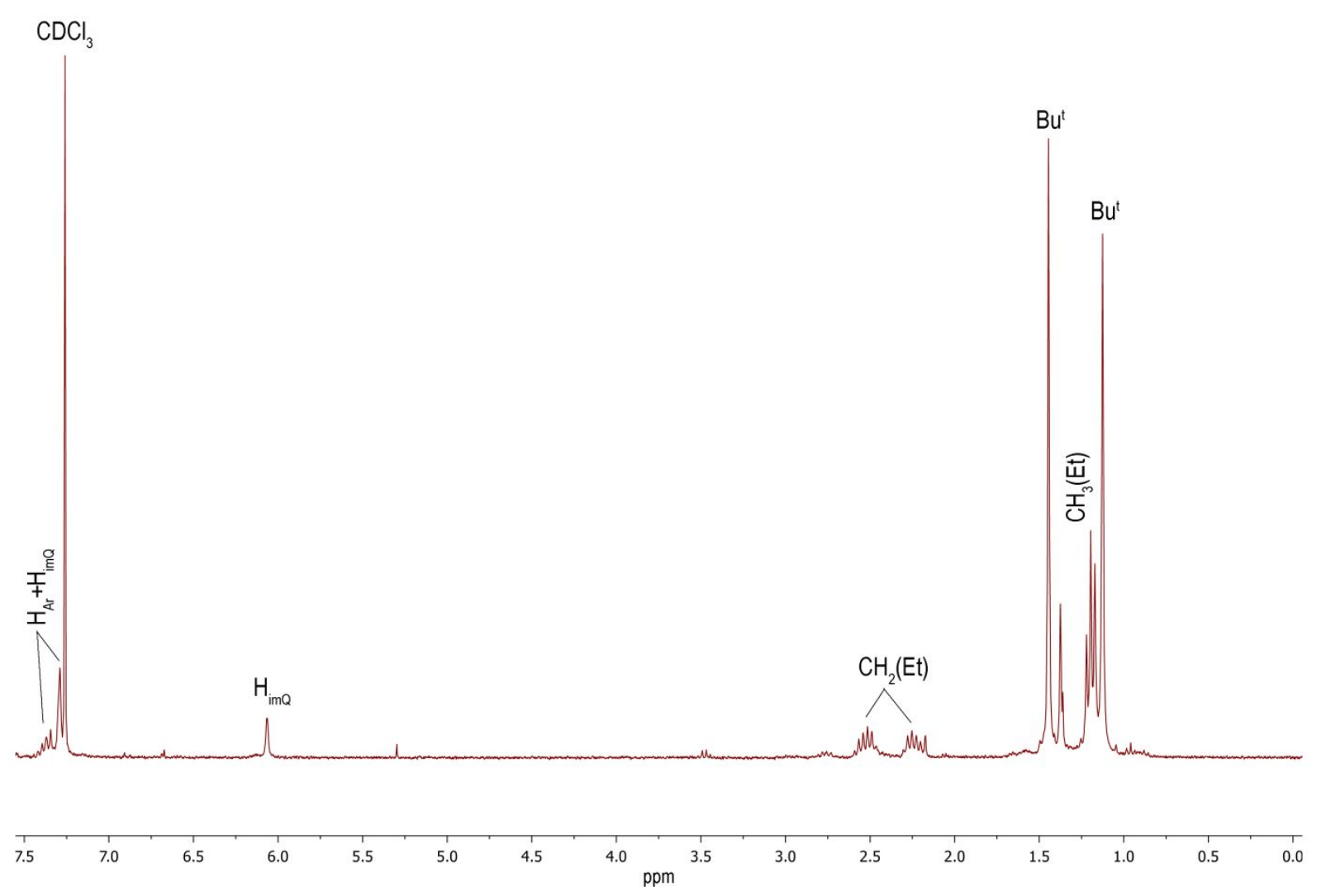

${ }^{1} \mathrm{H}$ NMR spectrum of $4 \cdot \operatorname{Inl}_{3}$ 


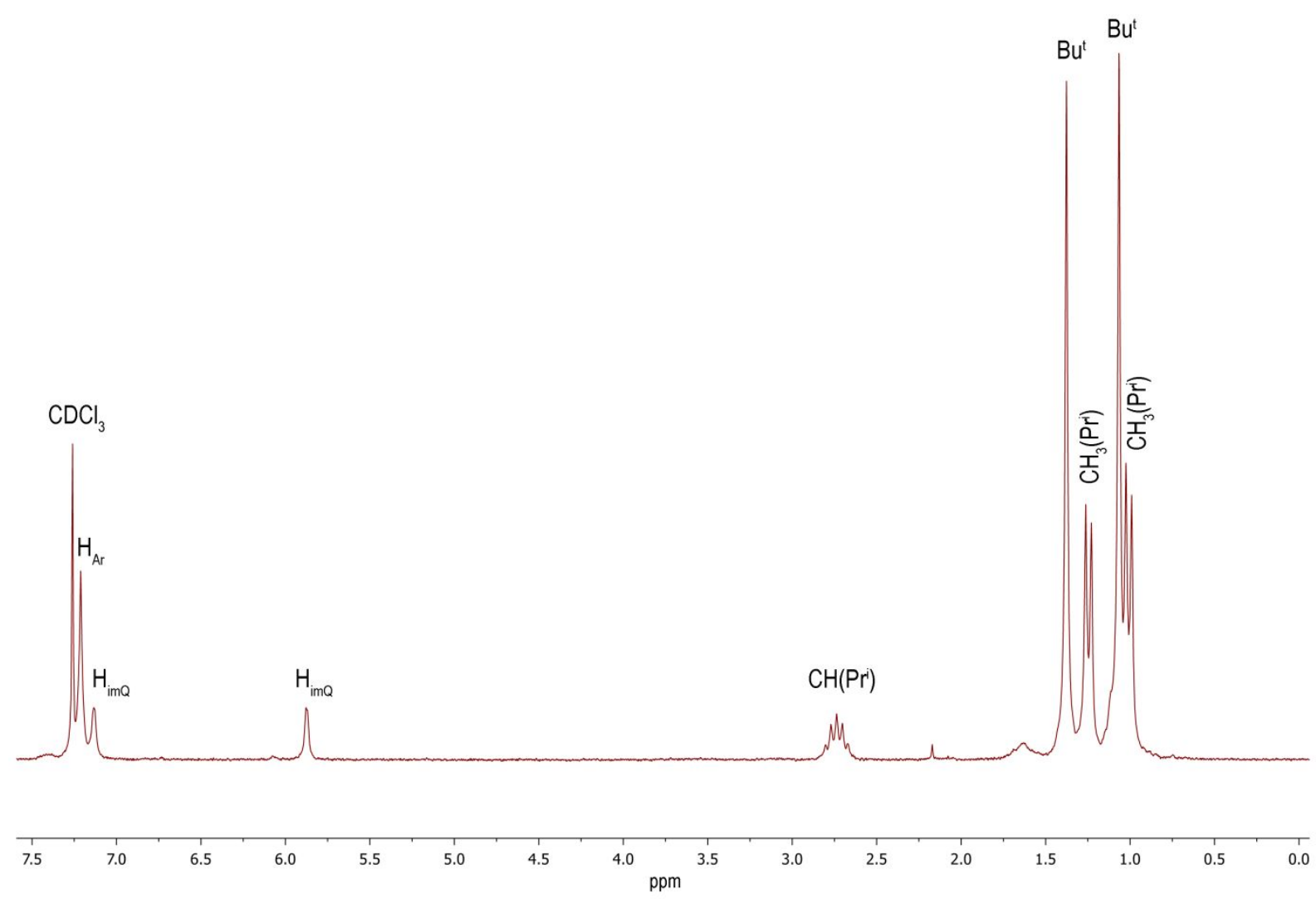

${ }^{1} \mathrm{H}$ NMR spectrum of $\mathbf{5} \cdot \mathrm{HgBr}_{2}$

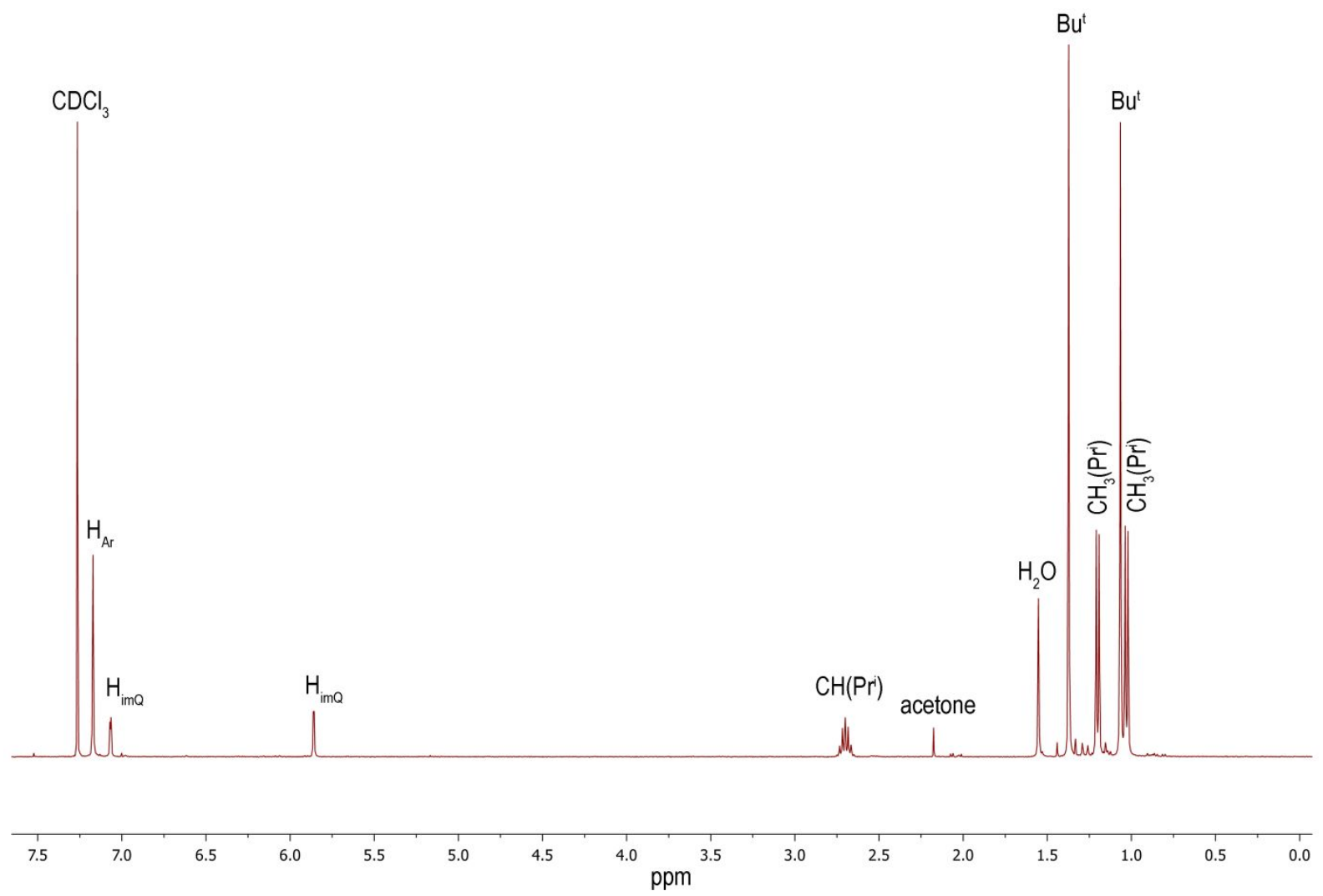

${ }^{1} \mathrm{H}$ NMR spectrum of $\mathbf{5} \cdot \mathrm{Hgl}_{2}$ 
Cyclic voltammograms of ligands 1-5

in $\mathrm{CH}_{2} \mathrm{Cl}_{2}$ solution, $0.1 \mathrm{M}\left[(\mathrm{n}-\mathrm{Bu})_{4} \mathrm{~N}\right] \mathrm{ClO}_{4}$, argon, $20^{\circ} \mathrm{C}$, Pt- working electrode

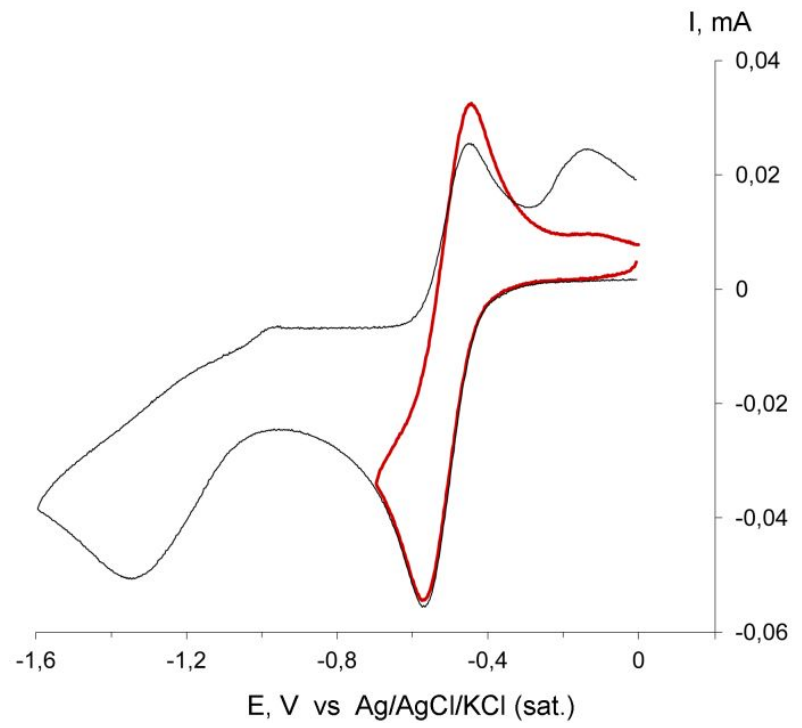

$\mathrm{CV}$ of 3,6-Q (1)

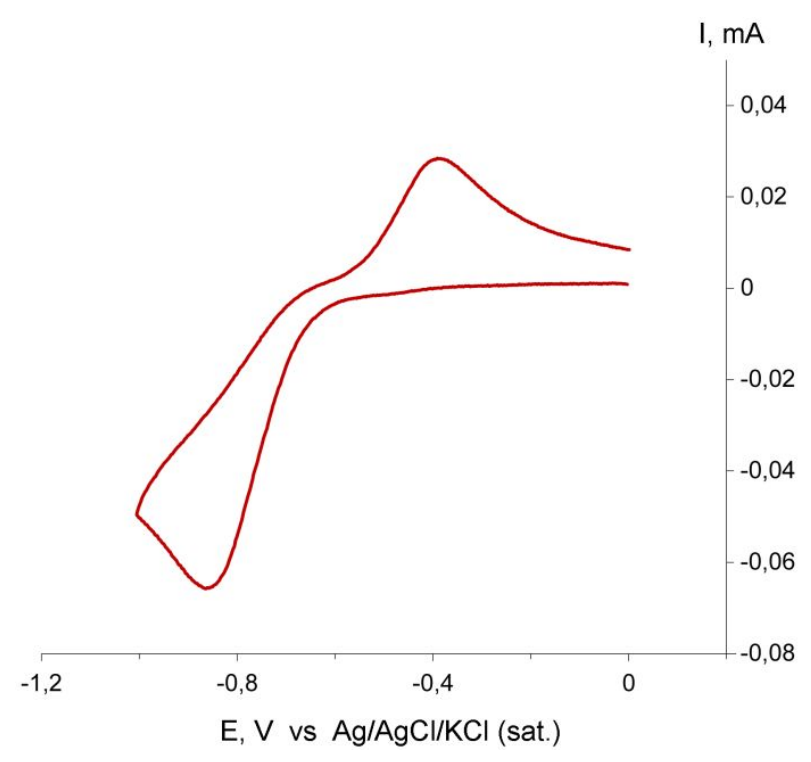

$\mathrm{CV}$ of imQMe (2) 


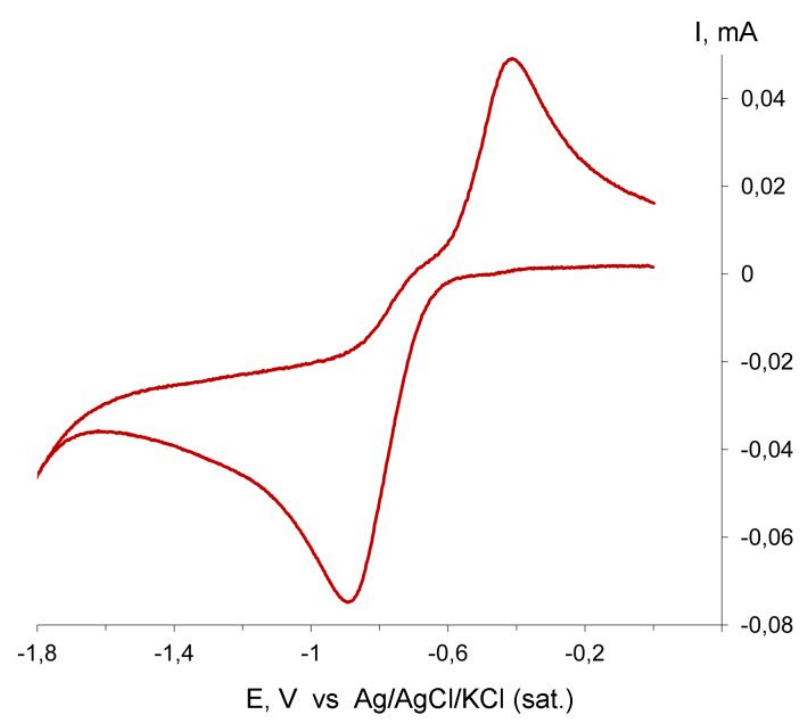

CV of imQMe,Et (3)

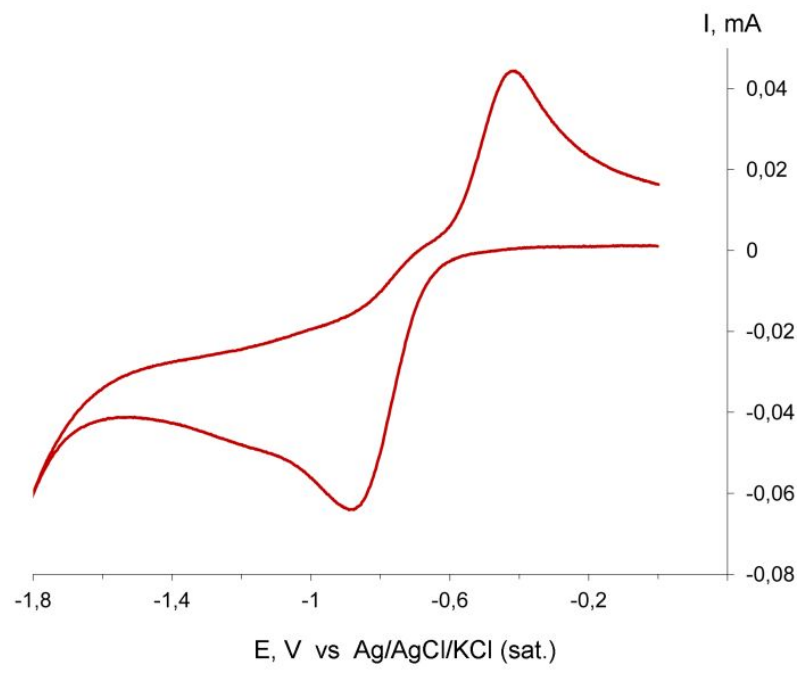

CV of imQ $Q^{\mathrm{Et}}(\mathbf{4})$

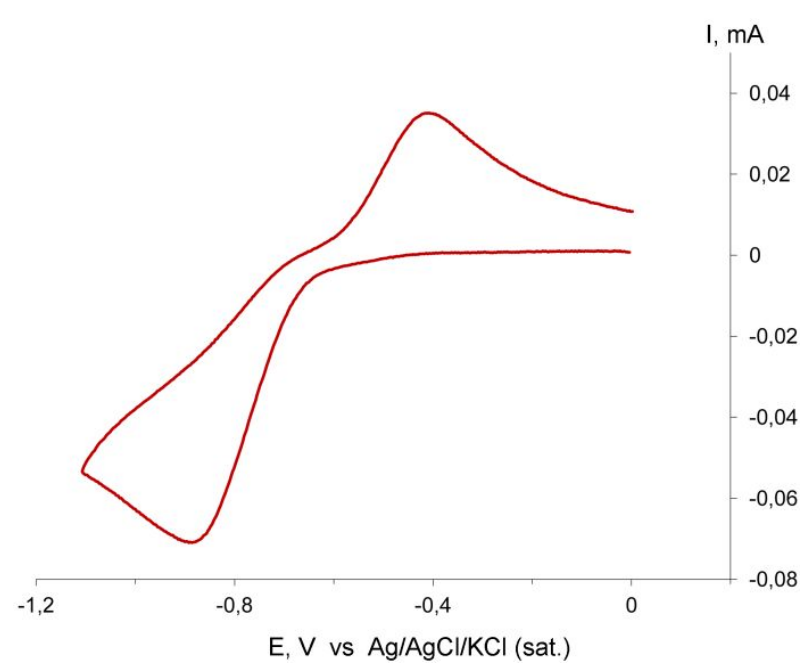

$\mathrm{CV}$ of $\mathrm{imQ} \mathrm{Q}^{\mathrm{i} P r}(\mathbf{5})$ 


\section{Cyclic voltammograms of complexes $1-5 \cdot M X_{n}$}

in $\mathrm{CH}_{2} \mathrm{Cl}_{2}$ solution, $0.1 \mathrm{M}\left[(\mathrm{n}-\mathrm{Bu})_{4} \mathrm{~N}\right] \mathrm{ClO}_{4}$, argon, $20^{\circ} \mathrm{C}$, Pt- working electrode

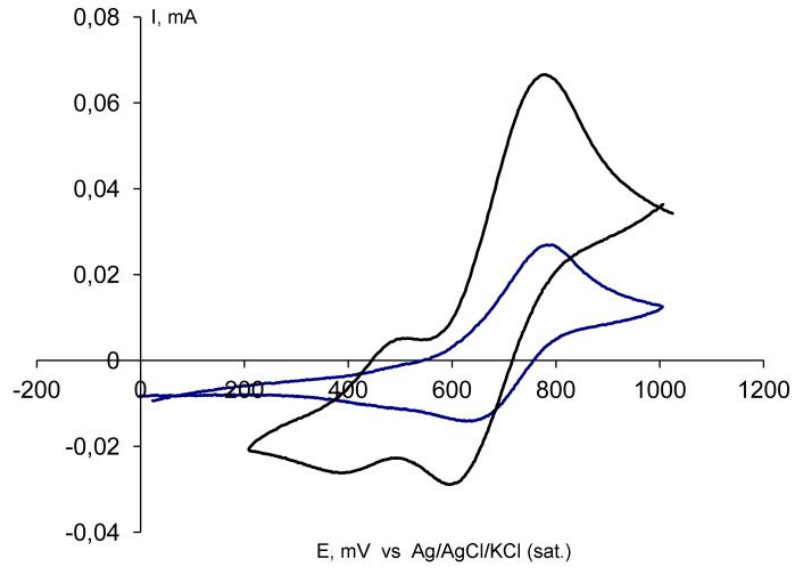

$\mathrm{CV}$ of $1 \cdot \mathrm{ZnBr}_{2}$

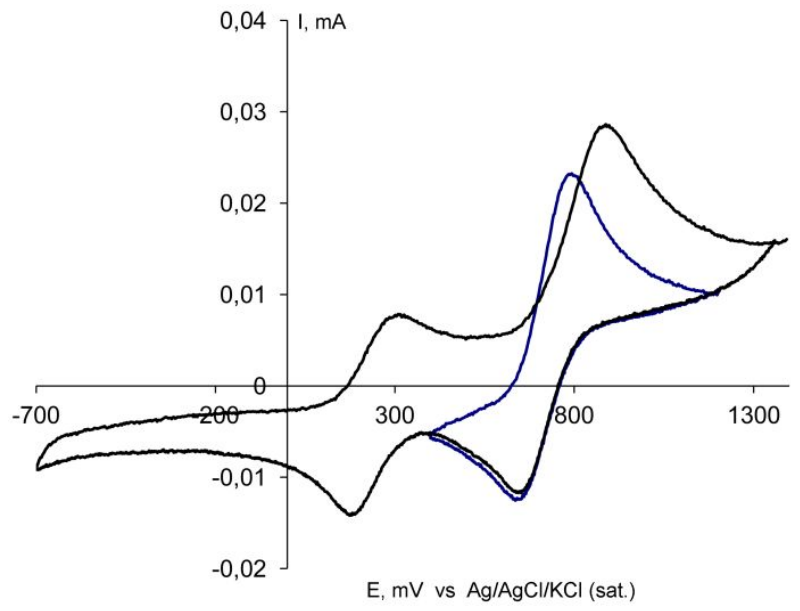

$\mathrm{CV}$ of $\mathbf{2} \cdot \mathrm{ZnI}_{2}$

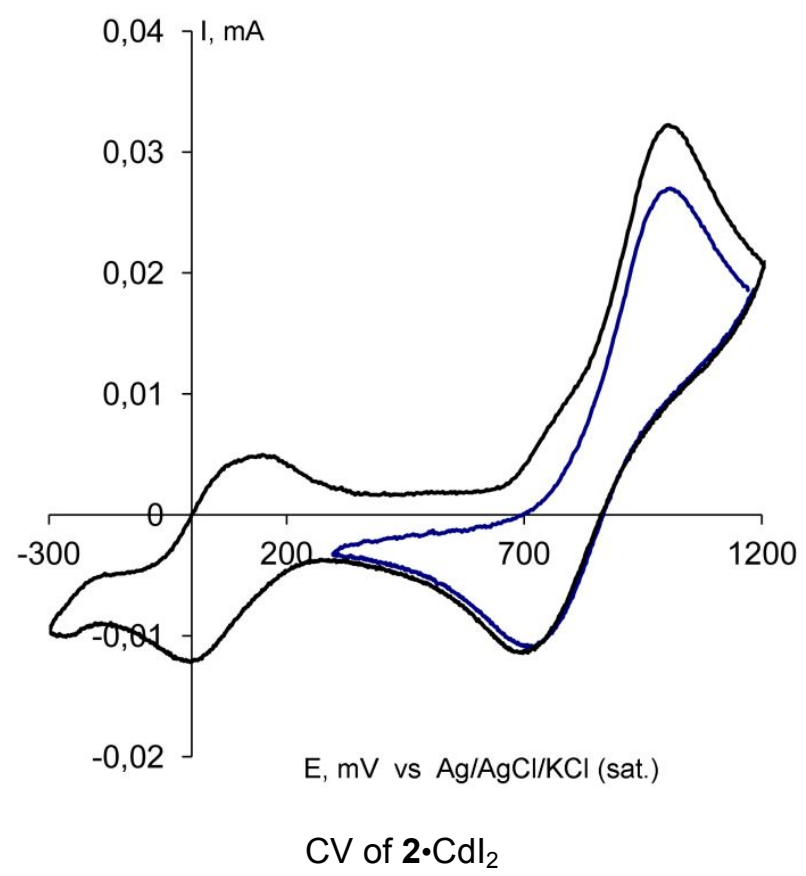




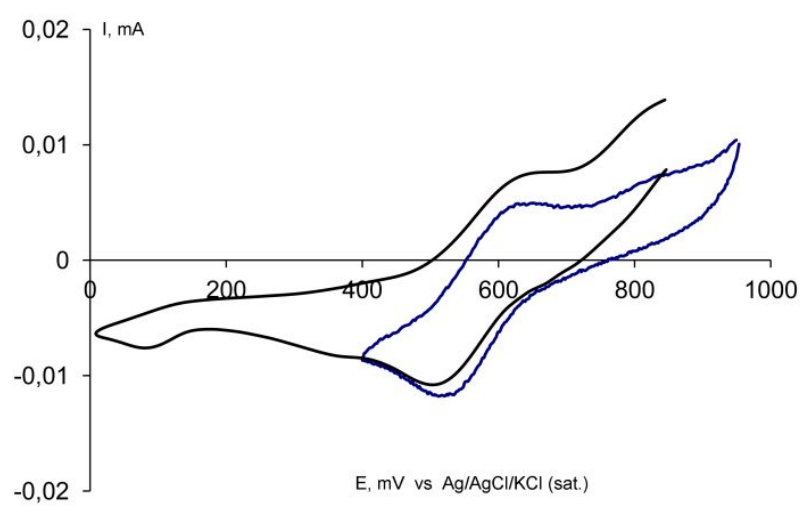

CV of $2 \cdot \operatorname{Inl}_{3}$

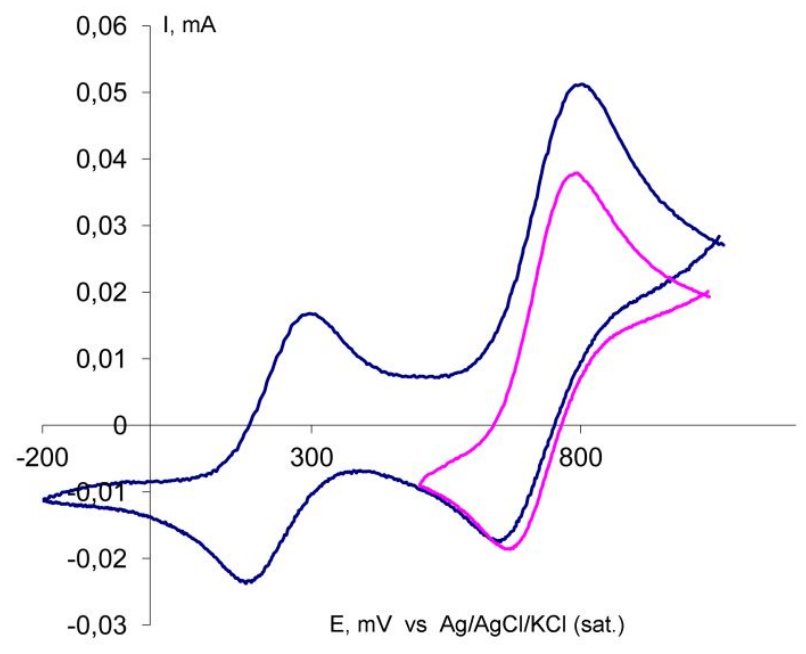

$\mathrm{CV}$ of $3 \cdot \mathrm{ZnI}_{2}$

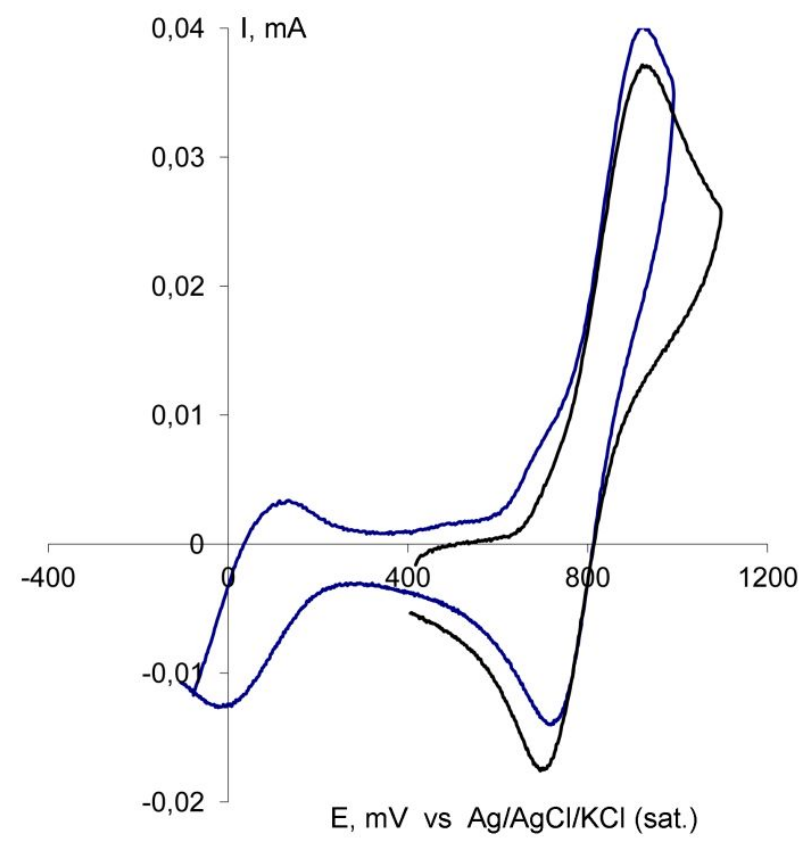

$\mathrm{CV}$ of $3 \cdot \mathrm{Cdl}_{2}$ 


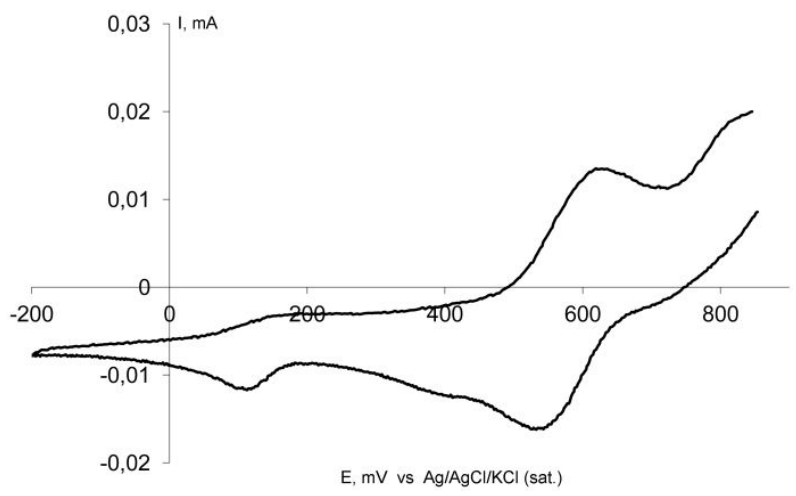

$\mathrm{CV}$ of 3·Inl 3

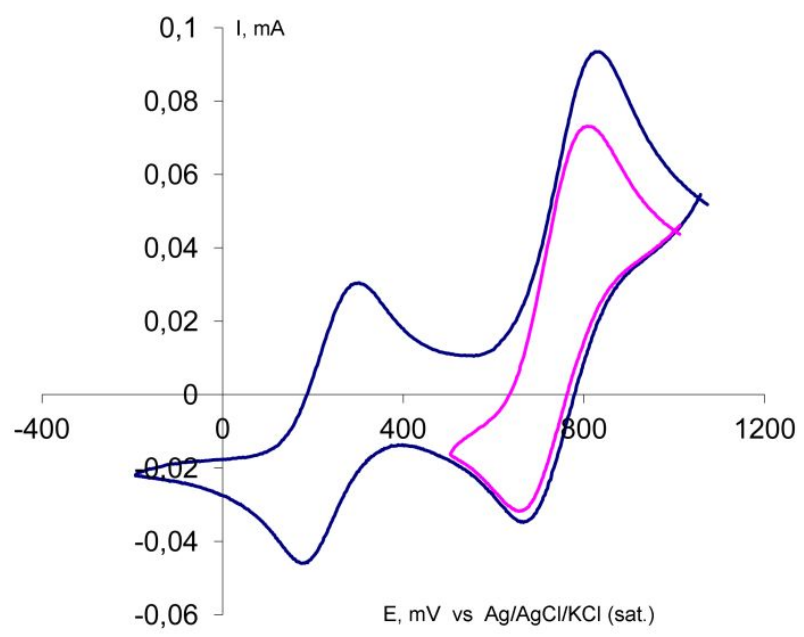

$\mathrm{CV}$ of $4 \cdot \mathrm{ZnI}_{2}$

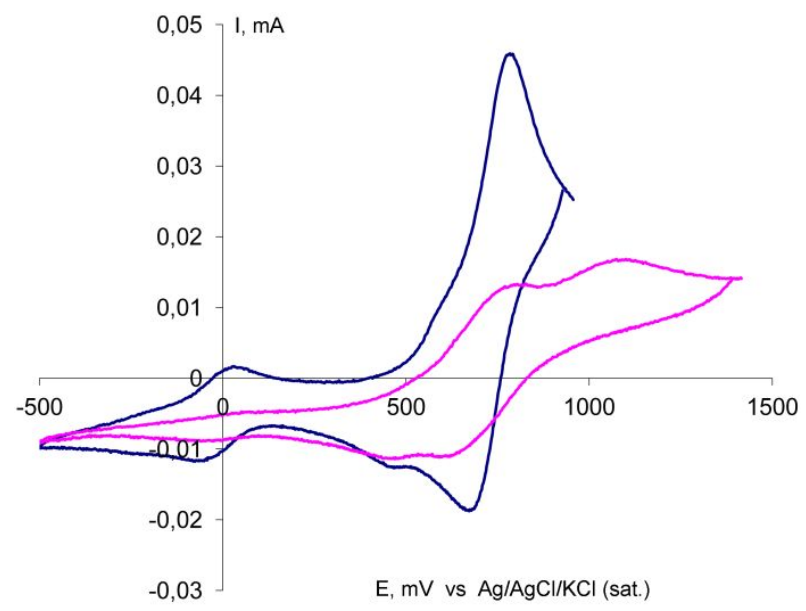

$\mathrm{CV}$ of $4 \cdot \mathrm{Cdl}_{2}$ (pink - in $\mathrm{CH}_{2} \mathrm{Cl}_{2}$, blue - in $\mathrm{CH}_{2} \mathrm{Cl}_{2}-\mathrm{CH}_{3} \mathrm{CN}$ mixture 1:1) 


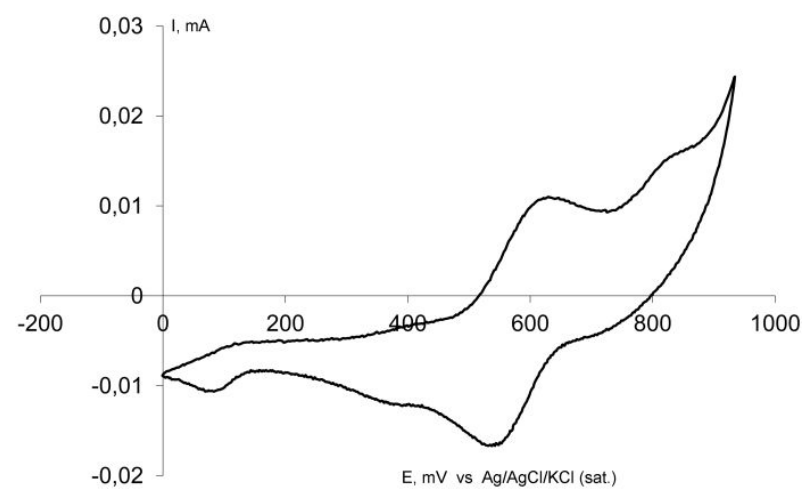

$\mathrm{CV}$ of $4 \cdot \operatorname{Inl}_{3}$

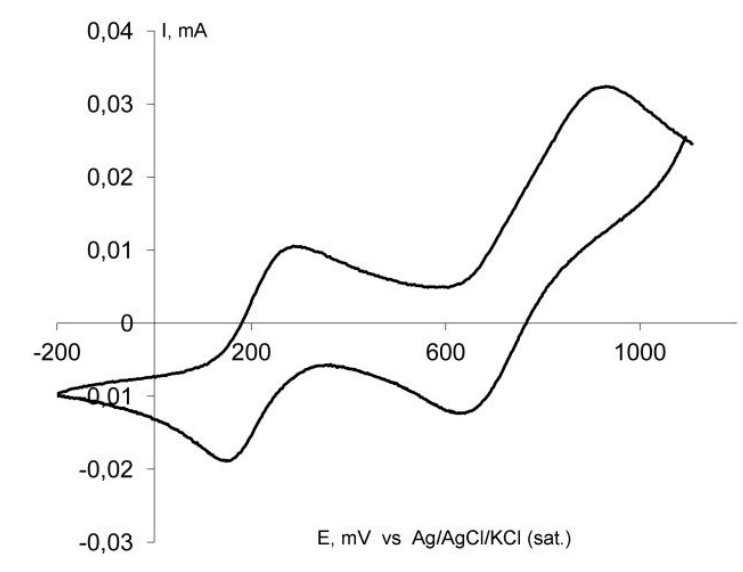

$\mathrm{CV}$ of $5 \cdot \mathrm{Znl}_{2}$

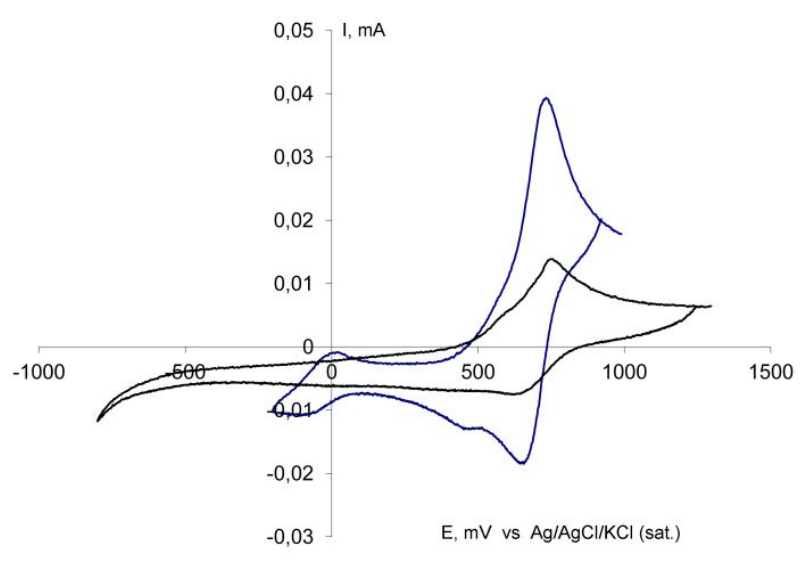

CV of $5 \cdot \mathrm{Cdl}_{2}$ (black - in $\mathrm{CH}_{2} \mathrm{Cl}_{2}$, blue - in $\mathrm{CH}_{2} \mathrm{Cl}_{2}-\mathrm{CH}_{3} \mathrm{CN}$ mixture 1:1)

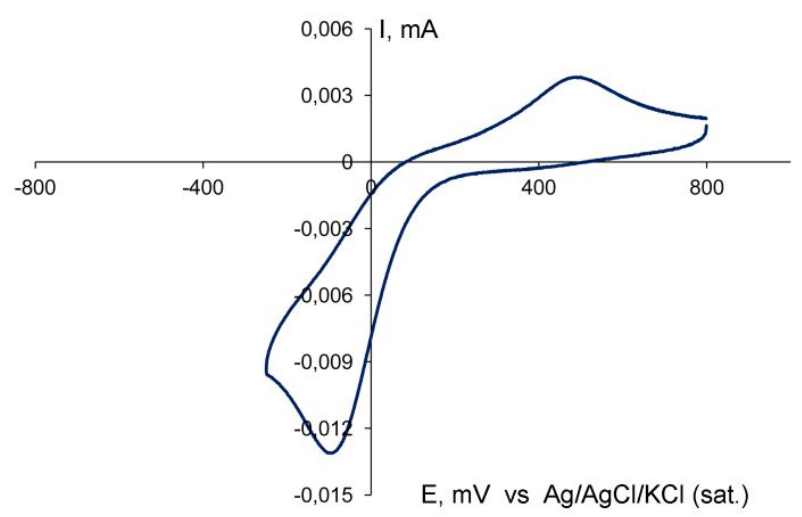

$\mathrm{CV}$ of $\mathbf{5} \cdot \mathrm{HgBr}_{2}$ 


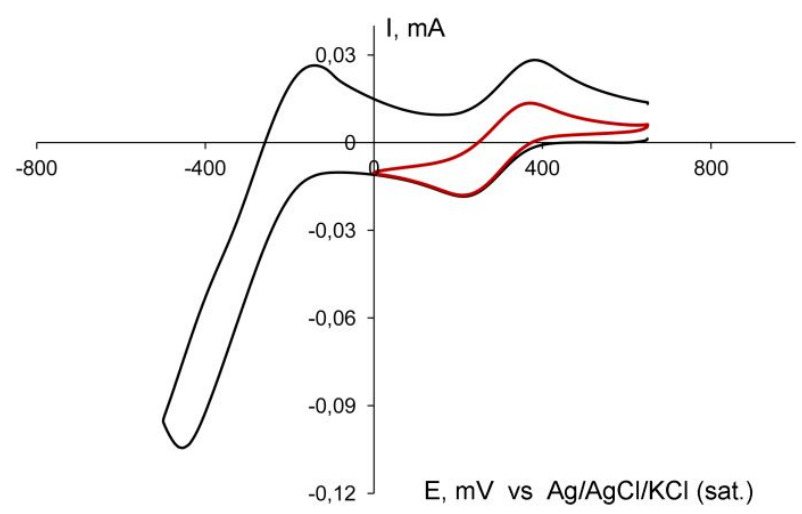

CV of $5 \cdot \mathrm{Hgl}_{2}$

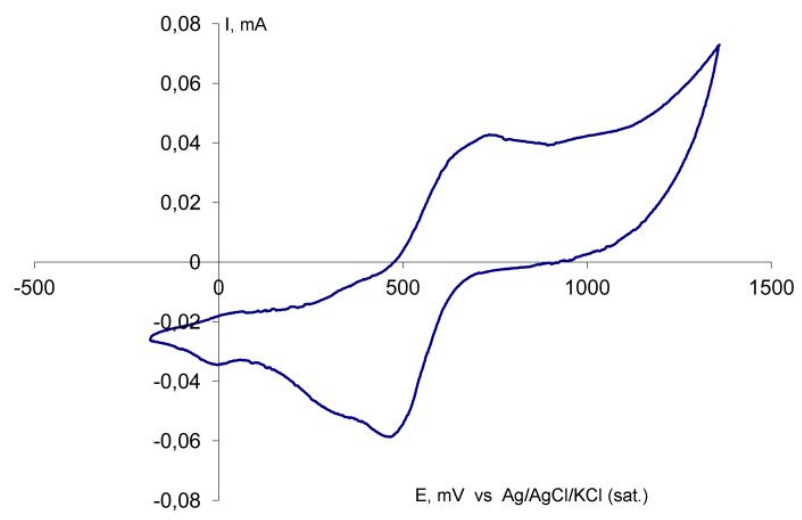

$\mathrm{CV}$ of $\mathbf{5} \cdot \operatorname{Inl}_{3}$ 


\section{References}

1. Perrin, D. D.; Armarego, W. L. F.; Perrin, D. R., Purification of Laboratory Chemicals. Pergamon Press: Oxford, 1980.

2. Garnov, V. A.; Nevodchikov, V. I.; Abakumova, L. G.; Abakumov, G. A.; Cherkasov, V. K. New tetrahydro-2, 3-naphthoquinones. Bull. Acad. Sci. USSR, Div. Chem. Sci. 1987, 36, 1728-1730.

3. Abakumov, G. A.; Druzhkov, N. O.; Kurskii, Y. A.; Shavyrin, A. S. NMR study of products of thermal transformation of substituted N-aryl-o-quinoneimines. Russ. Chem. Bull. Int. Ed. 2003, 52 (3), 712-717.

4. Poddel'sky, A. I.; Kurskii, Y. A.; Piskunov, A. V.; Somov, N. V.; Cherkasov, V. K.; Abakumov, G. A. Triphenylantimony $(\mathrm{V})$ o-amidophenolates with unsymmetrical $\mathrm{N}$-aryl group for a reversible dioxygen binding Appl. Organomet. Chem. 2011, 25 (3), 180-189.

5. Gynane, M. J. S.; Wilkinson, M.; Worrall, I. J. A new facile synthesis of indium(III) iodide. Inorg. Nucl. Chem. Lett. 1973, 9, 765-766.

6. Abakumov, G. A.; Cherkasov, V. K.; Piskunov, A. V.; Meshcheryakova, I. N.; Maleeva, A. V.; Poddel'skii, A. I.; Fukin, G. K. Zinc Molecular Complexes with Sterically Hindered o-Quinone and oIminoquinone. Dokl. Chem. 2009, 427, 168-171.

7. Piskunov, A. V.; Mescheryakova, I. N.; Bogomyakov, A. S.; Romanenko, G. V.; Cherkasov, V. K.; Abakumov, G. A. Novel indium(III) complexes with sterically hindered o-iminobenzoquinone. Inorg. Chem. Commun. 2009, 12 (10), 1067-1070.

8. $\quad$ Bruker (2012). Smart. Bruker AXS Inc., Madison, Wisconsin, USA.

9. $\quad$ Bruker (2018). APEX3. Bruker AXS Inc., Madison, Wisconsin, USA.

10. Rigaku Oxford Diffraction. (2015). CrysAlis ${ }^{\text {Pro }}$ software system, ver. 1.171.38.46, Rigaku Corporation, Wroclaw, Poland.

11. Sheldrick, G. M. Crystal Structure Refinement with SHELXL. Acta Cryst. 2015, C71, 3-8.

12. Krause, L.; Herbst-Irmer, R.; Sheldrick, G. M.; Stalke, D. Comparison of silver and molybdenum microfocus X-ray sources for single-crystal structure determination. J. Appl. Cryst. 2015, 48, 3-10.

13. Frisch, M. J.; Trucks, G. W.; Schlegel, H. B.; Scuseria, G. E.; Robb, M. A.; Cheeseman, J. R.; Scalmani, G.; Barone, V.; Mennucci, B.; Petersson, G. A.; Nakatsuji, H.; Caricato, M.; Li, X.; Hratchian, H. P.; Izmaylov, A. F.; Bloino, J.; Zheng, G.; Sonnenberg, J. L.; Hada, M.; Ehara, M.; Toyota, K.; Fukuda, R.; Hasegawa, J.; Ishida, M.; Nakajima, T.; Honda, Y.; Kitao, O.; Nakai, H.; Vreven, T.; Montgomery, J. A.; Jr., J. E. P.; Ogliaro, F.; Bearpark, M.; Heyd, J. J.; Brothers, E.; Kudin, K. N.; Staroverov, V. N.; Keith, T.; Kobayashi, R.; Normand, J.; Raghavachari, K.; Rendell, A.; Burant, J. C.; Iyengar, S. S.; Tomasi, J.; Cossi, M.; Rega, N.; Millam, J. M.; Klene, M.; Knox, J. E.; Cross, J. B.; Bakken, V.; Adamo, C.; Jaramillo, J.; Gomperts, R.; Stratmann, R. E.; Yazyev, O.; Austin, A. J.; Cammi, R.; Pomelli, C.; Ochterski, J. W.; Martin, R. L.; Morokuma, K.; Zakrzewski, V. G.; Voth, G. A.; Salvador, P.; Dannenberg, J. J.; Dapprich, S.; Daniels, A. D.; Farkas, O.; Foresman, J. B.; Ortiz, J. V.; Cioslowski, J.; Fox, D. J. Gaussian 09 (Revision E.01), Wallingford CT, Gaussian 09 (Revision E.01), Gaussian, Inc., Wallingford CT, 2013.

14. Becke, A. D. Density-functional thermochemistry. III. The role of exact exchange. J. Chem. Phys. 1993, 98, 5648-5652.

15. Brown, S. N. Metrical Oxidation States of 2-Amidophenoxide and Catecholate Ligands: Structural Signatures of Metal-Ligand $\pi$ Bonding in Potentially Noninnocent Ligands. Inorg. Chem. 2012, 51, 12511260.

16. Fokin, S. V.; Fursova, E. Y.; Letyagin, G. A.; Bogomyakov, A. S.; Morozov, V. A.; Romanenko, G. V.; Ovcharenko, V. I. Structure and Magnetic Properties of Mixed-Ligand Complexes of 3d Metal Hexafluoroacetylacetonates with 3,5- and 3,6-Di-Tert-Butyl-O-Benzoquinones. J. Struct. Chem. 2020, 61, 541-549.

17. Maity, S.; Kundu, S.; Bera, S.; Weyhermüller, T.; Ghosh, P. o-Iminobenzoquinone and o-Iminobenzosemiquinonate Anion Radical Complexes of Rhodium and Ruthenium Eur. J. Inorg. Chem. 2016, 2016 (22), 3691-3697.

18. Mondal, M. K.; Mukherjee, C. An unprecedented one-step synthesis of octahedral Cu(II)bis(iminoquinone) complexes and their reactivity with $\mathrm{NaBH}_{4}$. Dalton Trans. 2016, 45 (34), 13532-13540. 19. Paul, G. C.; Das, K.; Maity, S.; Begum, S.; Srivastava, H. K.; Mukherjee, C. Geometry-Driven Iminosemiquinone Radical to $\mathrm{Cu}(\mathrm{II})$ Electron Transfer and Stabilization of an Elusive Five-Coordinate 
$\mathrm{Cu}(\mathrm{I})$ Complex: Synthesis, Characterization, and Reactivity with $\mathrm{KO}_{2}$. Inorg. Chem. 2019, 58 (3), 17821793.

20. Coughlin, E. J.; Zeller, M.; Bart, S. C. Neodymium(III) Complexes Capable of Multi-Electron Redox Chemistry. Angew. Chem. Int. Ed. 2017, 56 (40), 12142-12145.

21. Sinitsa, D. K.; Sukhikh, T. S.; Konchenko, S. N.; Pushkarevsky, N. A. Synthesis, structures, and oneor two-electron reduction reactivity of mononuclear lanthanide (Ho, Dy) complexes with sterically hindered o-iminobenzoquinone ligands. Polyhedron 2021, 195, 114967.

22. Coughlin, E. J.; Qiao, Y.; Lapsheva, E.; Zeller, M.; Schelter, E. J.; Bart, S. C. Uranyl Functionalization Mediated by Redox-Active Ligands: Generation of O-C Bonds via Acylation. J. Am. Chem. Soc. 2019, 141 (2), 1016-1026. 